\title{
UDC 004.9
}

SCOPUS CODE 1705

https://doi.org/10.36073/1512-0996-2020-2-24-49

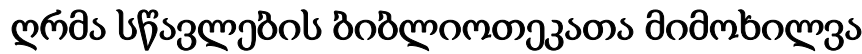

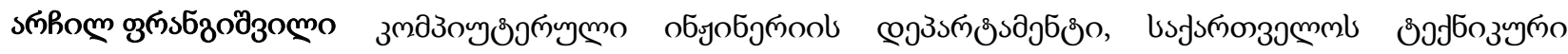

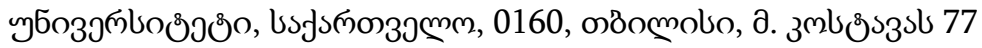 \\ E-mail: a_prangi@gtu.ge

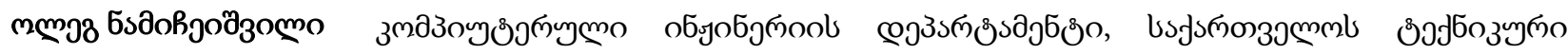

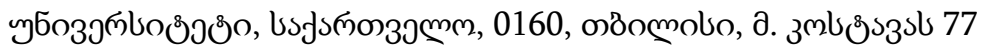 \\ E-mail: o.namicheishvili@gtu.ge

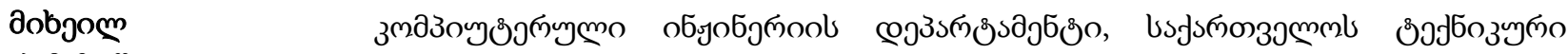

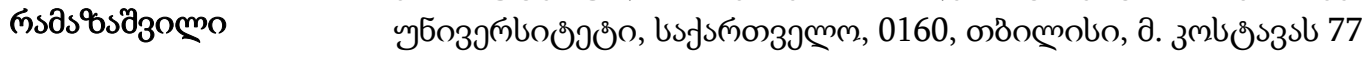 \\ E-mail: m.ramazashvili@gtu.ge
}

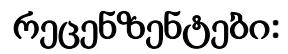

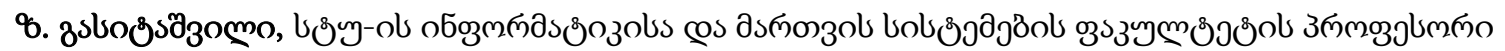

E-mail: zur_gas@gtu.ge

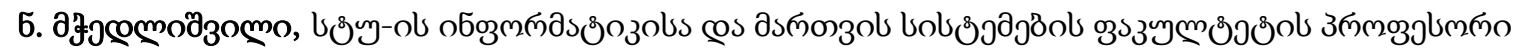

E-mail: galoba47@mail.ru

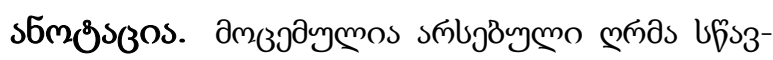

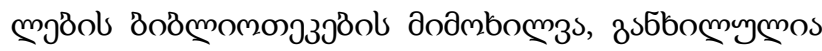

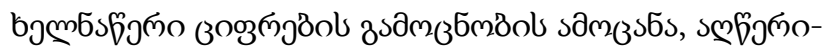

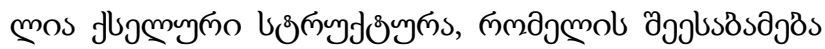

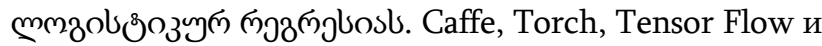

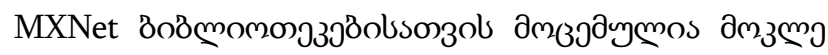

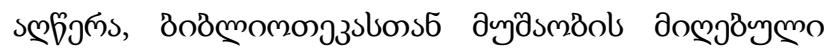

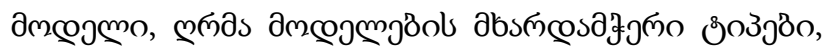

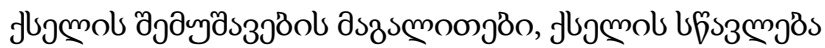

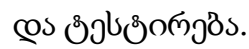

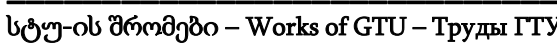

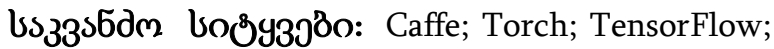

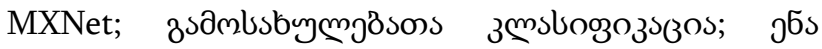

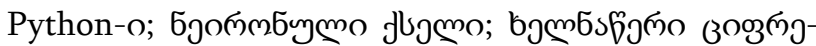

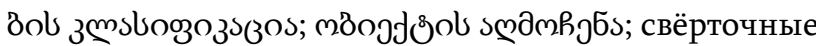
слои; обучающая машина.

\section{gjlsşsmo}

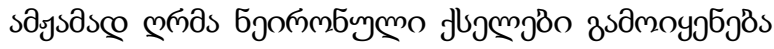

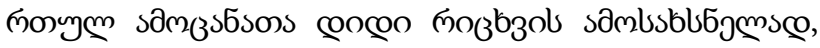

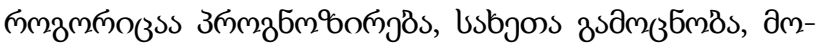

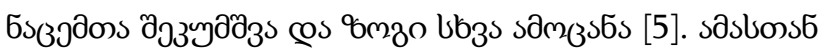




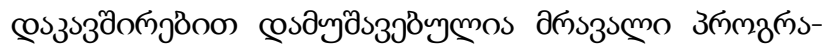

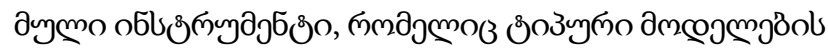

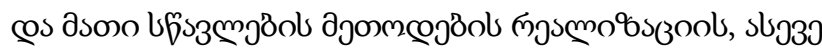

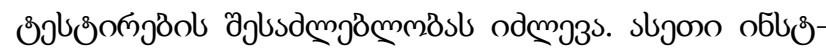

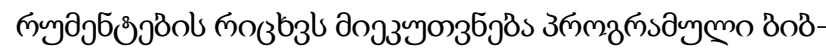

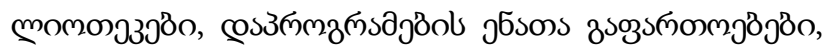

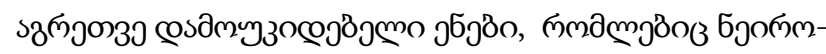

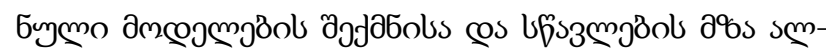

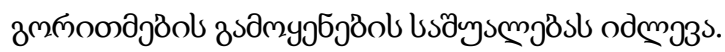

\section{domoossen Esformo}

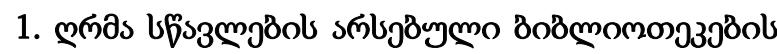
додмьомзз

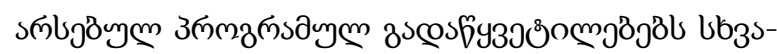

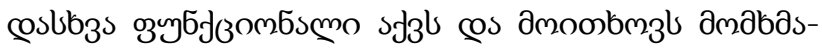

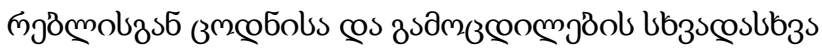

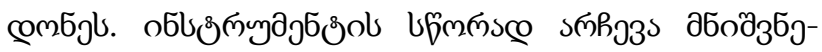

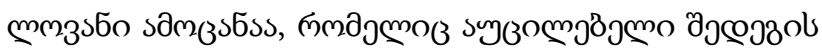

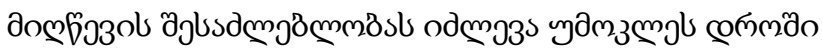

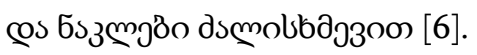

јзадмо (збпомо 1) амузьбомпо обозмпазъо

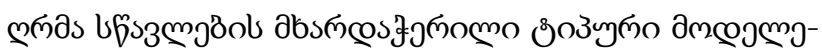

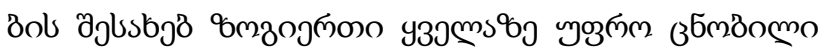

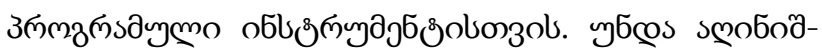

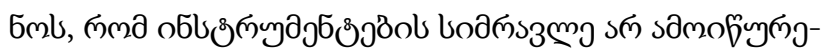

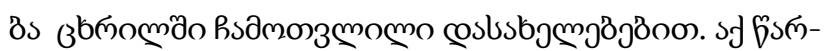

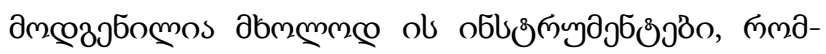

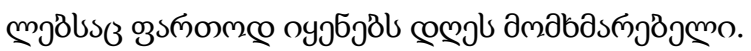

उb̆омо 1

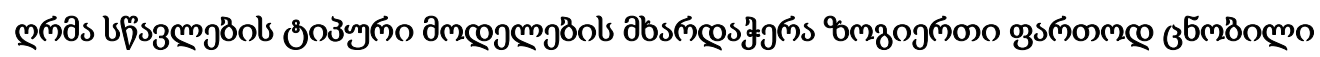

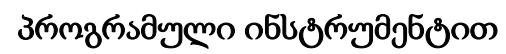

\begin{tabular}{|c|c|c|c|c|c|c|c|c|}
\hline № & costusbjemgass & 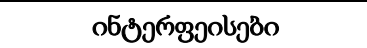 & 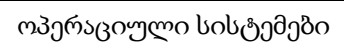 & FCNN & CNN & RNN & $\mathrm{AE}^{1}$ & $\mathrm{RBM}^{2}$ \\
\hline 1 & Caffe[7] & C++, Python, MATLAB & Linux, Windows, OSX & + & + & + & + & - \\
\hline 2 & Torch $[8]$ & C,Lua & Linux, iOS, Android & + & + & + & + & + \\
\hline 3 & TensorFlow[9] & Python, Java,Go & $\begin{array}{l}\text { Linux, Windows, MacOS, } \\
\text { Android }\end{array}$ & + & + & + & + & + \\
\hline 4 & $\mathrm{MXNet}^{3[10]}$ & $\begin{array}{l}\text { C++, Python, R, Scala, Julia, } \\
\text { Perl, MATLAB, JavaScript }\end{array}$ & Linux, Windows, MacOS & + & + & + & + & + \\
\hline 5 & $\begin{array}{l}\text { Intel®neon }^{\mathrm{TM}} \\
\text { Framework [11] }\end{array}$ & Python & Linux, MacOS & + & + & + & + & - \\
\hline 6 & PyTorch[12] & Python & Linux, Windows, MacOS & + & + & + & + & - \\
\hline 7 & Theano $4[13]$ & Python & Linux, Windows, MacOS & + & + & +4 & + & + \\
\hline 8 & $\operatorname{Keras}^{5[14]}$ & Python & Linux,Vagrant & + & + & + & + & + \\
\hline 9 & Deepnet $^{6[15]}$ & Python & Linux & + & + & - & + & + \\
\hline 10 & Deepmat $7[16]$ & MATLAB & $\begin{array}{l}\text { Linux, Windows, MacOS, } \\
\text { Solaris }\end{array}$ & + & + & - & + & + \\
\hline 11 & $\operatorname{Darch}^{8[17]}$ & $\mathrm{R}$ & Linux, Windows & + & - & - & + & + \\
\hline
\end{tabular}




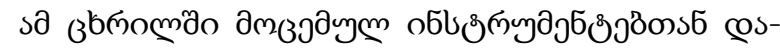

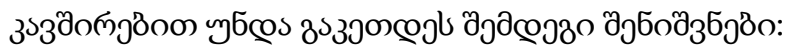

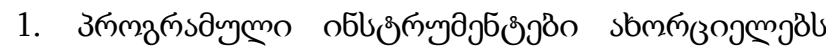

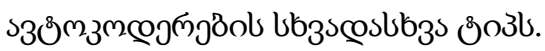

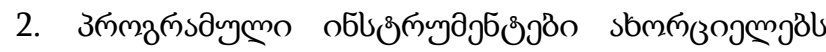

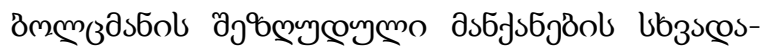

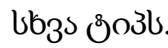

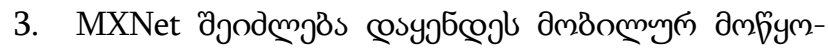

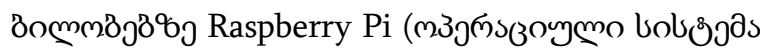

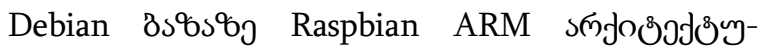

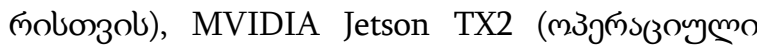
bob(̊as Ubuntu Arch64 8s\%s\%j).

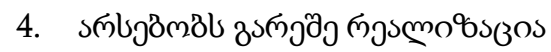

[http://www.wildml.com/2015/09/recurrent-neuralnetworks-tutorial-part-2-implementing-a-languagemodel-rnn-with-python-numpy-and-theano]/ ]

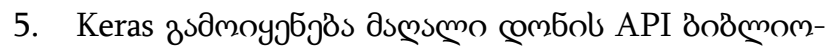
๓эзgB̊obsonzol TensorFlow, CNTK s6 Theano.

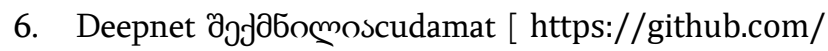
cudamat/cudamat] @os cuda-convnet [https:// code.google.com/archive/p/cuda-convnet/]

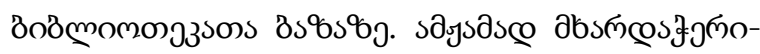
mo sल smols.

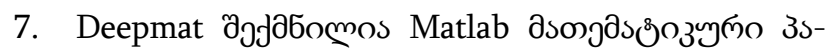

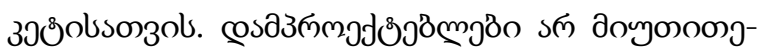

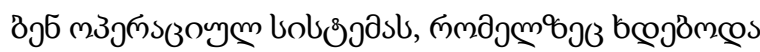

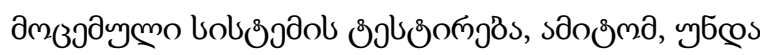

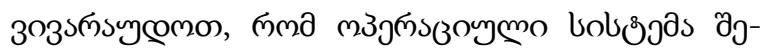

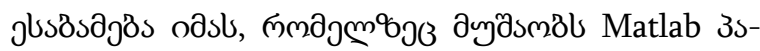

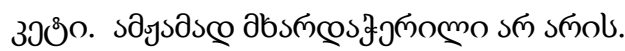

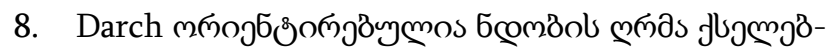

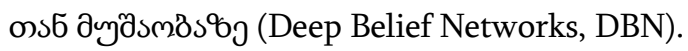

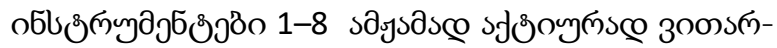

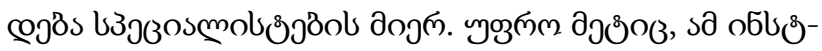

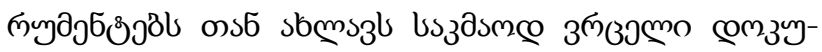

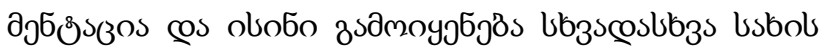

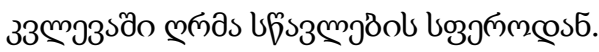

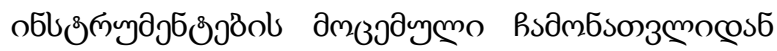

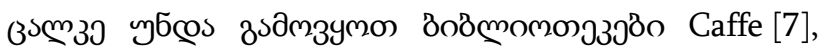
Torch [8], TensorFlow [9] @os MXNet [10].

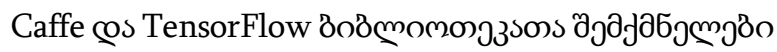

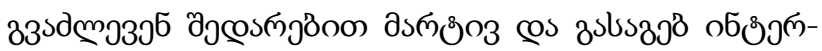

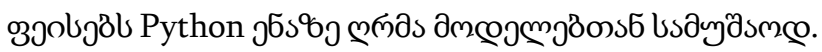

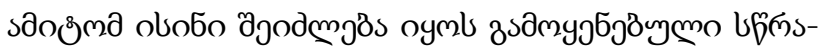

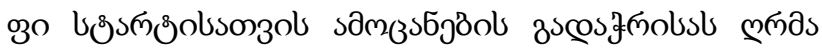

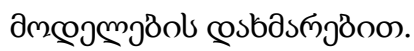

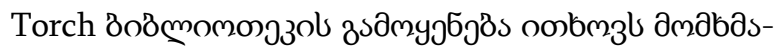

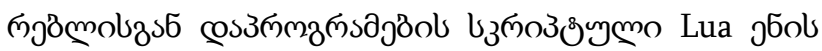

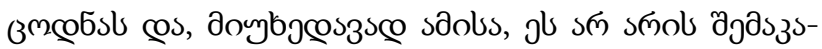

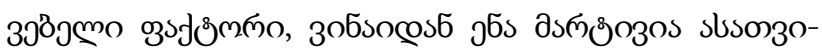

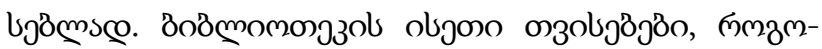

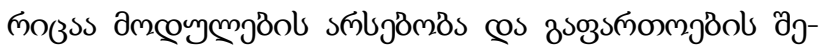

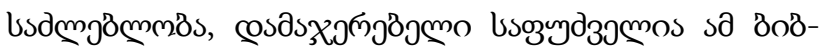

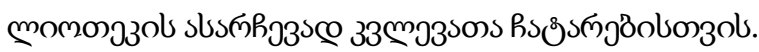

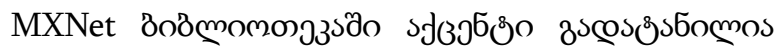

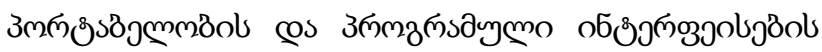

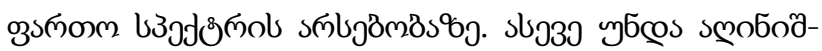

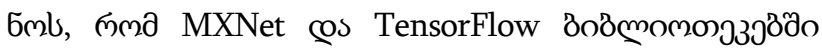

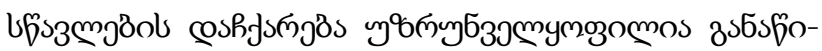

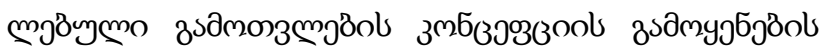

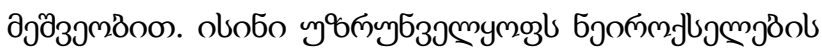

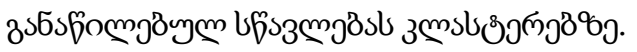

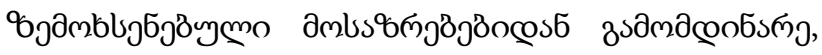

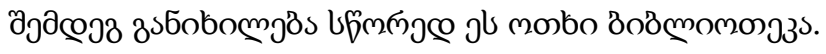




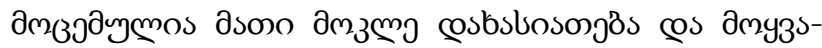

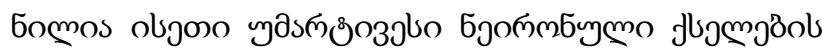

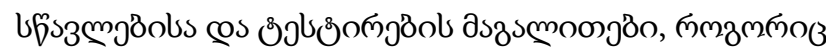

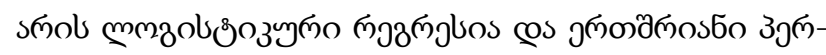

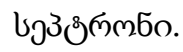

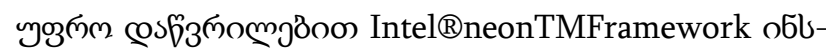

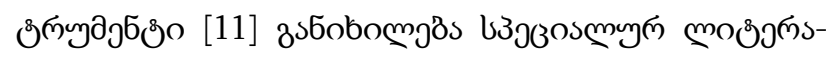

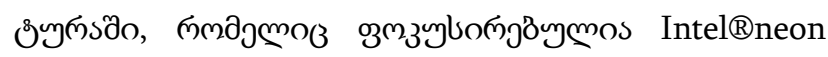

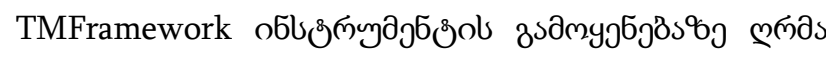

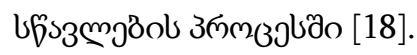

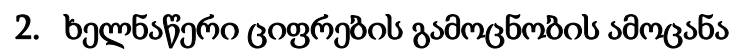

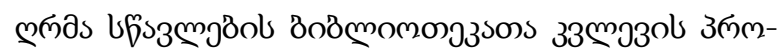

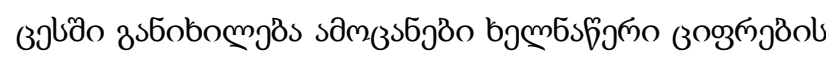

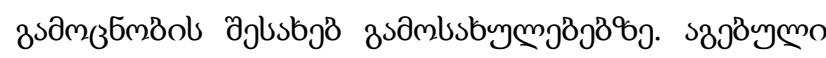

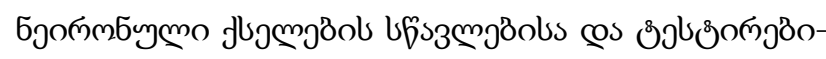

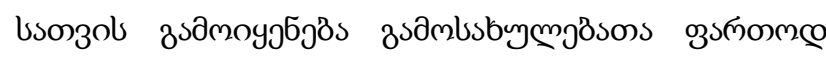

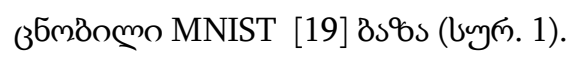

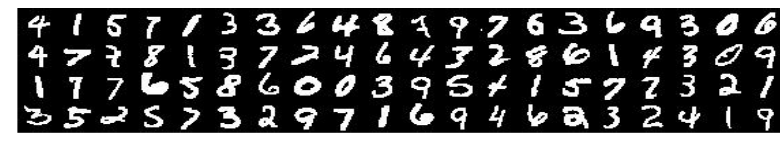

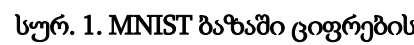

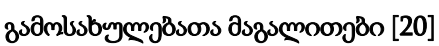

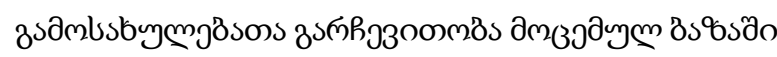

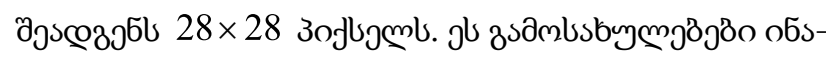

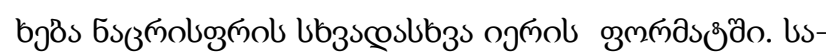

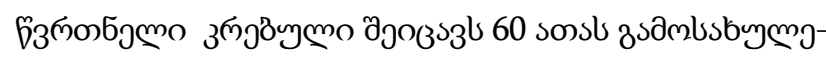

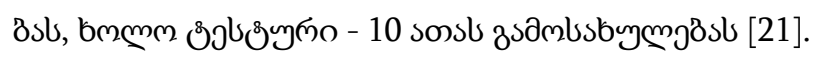

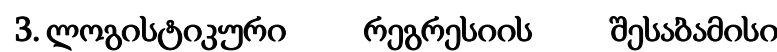

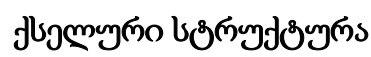

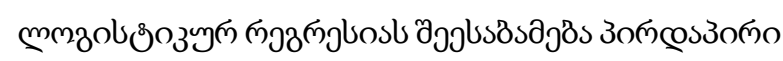

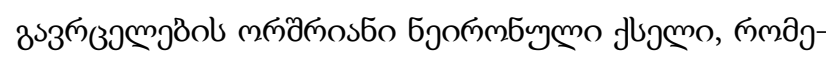

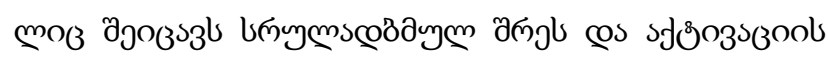

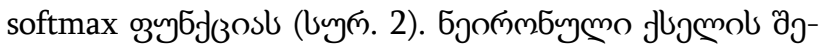

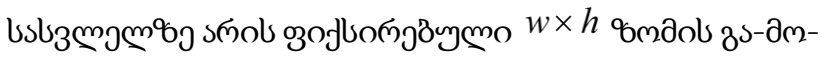

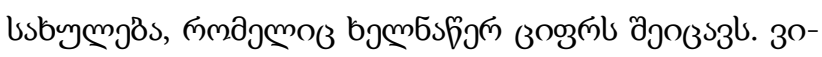

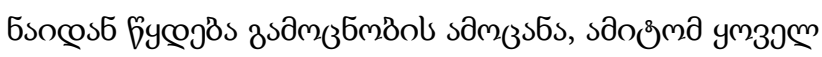

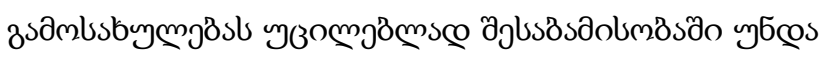

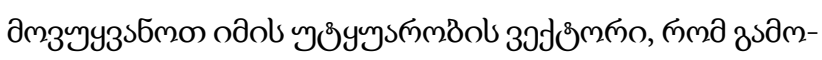

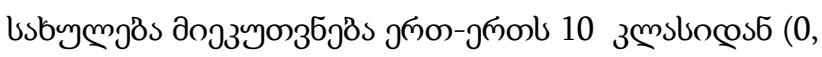

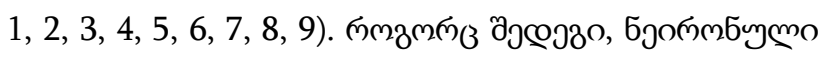

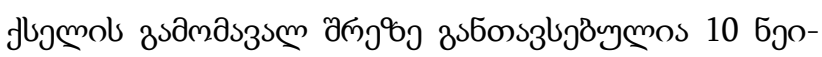

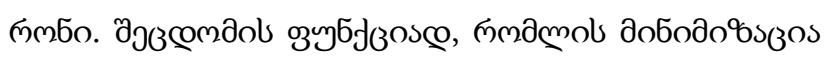

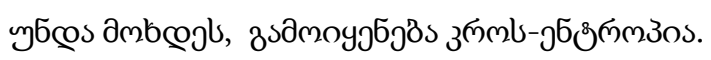

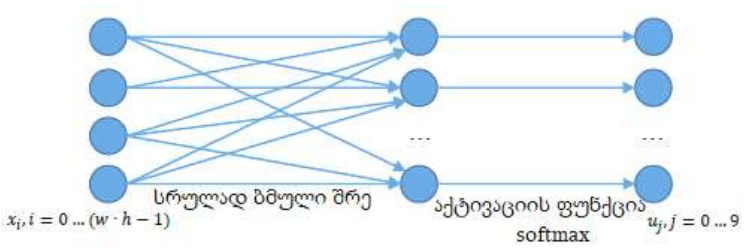

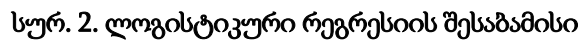

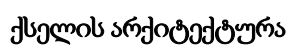

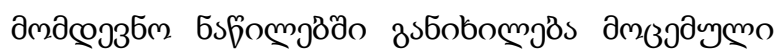

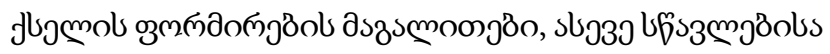

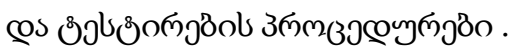

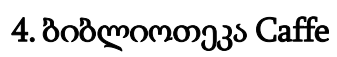

\section{1 дмзм⿻ sмб}

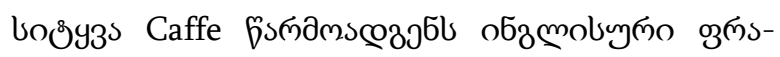

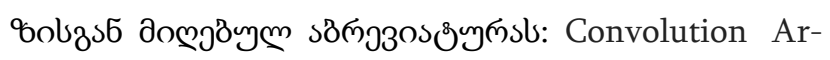
chitecture For Feature Extraction - зм5

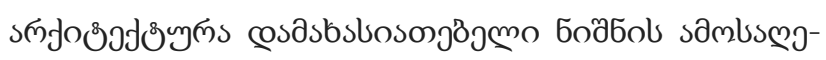

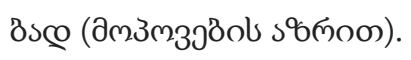

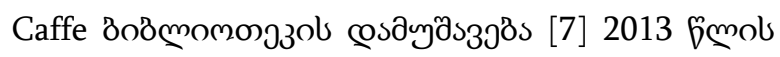

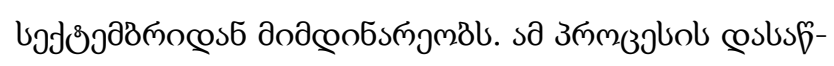

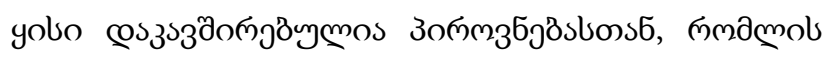




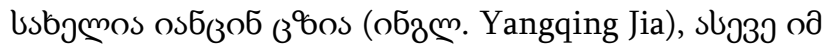

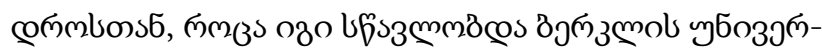

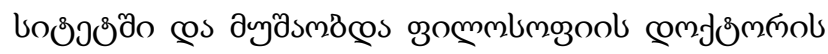

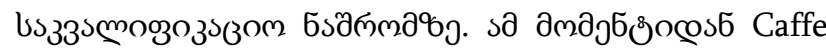

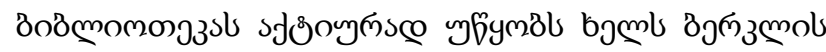

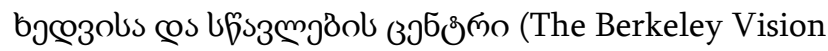

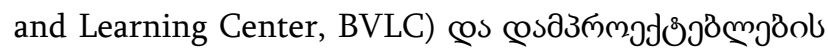

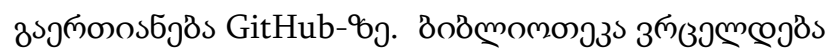
BSD 2-Clause-ob mo(3j6\%ono [21].

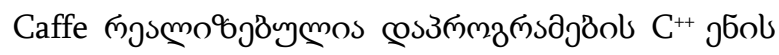

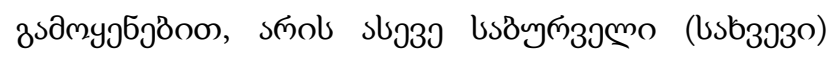
Python-\%g es MATLAB-\%g. mozozosmymse ozo

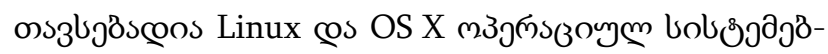

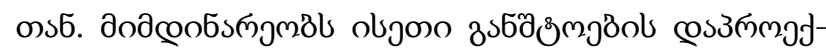

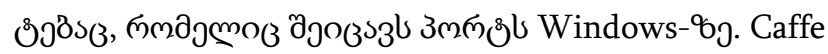
oyj6jßl BLAS (ATLAS, INTEL MKL, OpenBLAS)

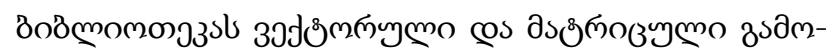

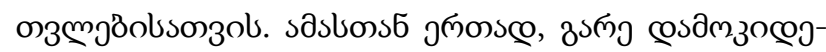

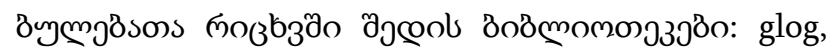
gflags, OpenCV, ptoroBuf, boost, snappy, hdfs, lmdb.

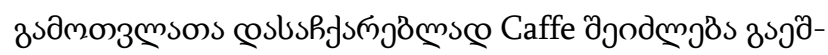

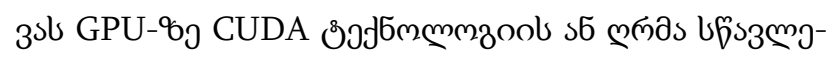

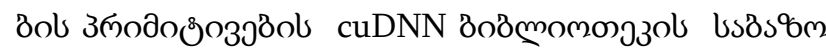
әృ

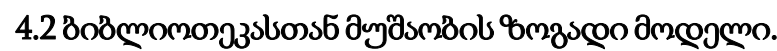

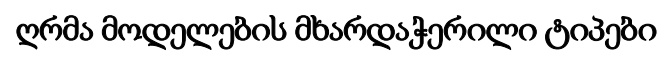

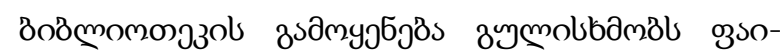

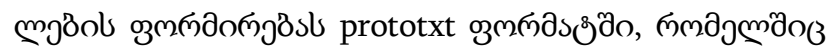

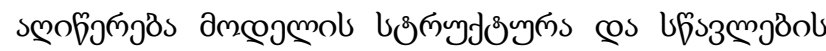

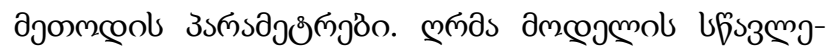

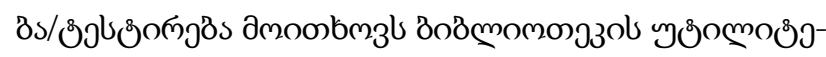

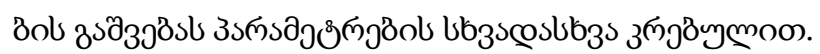

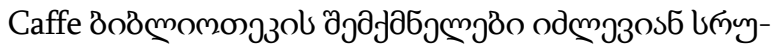

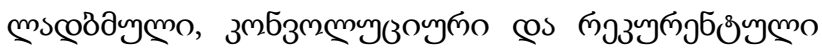

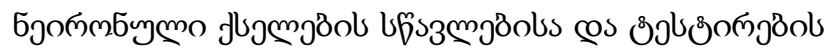

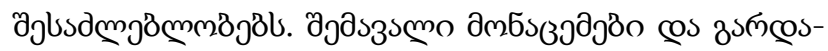

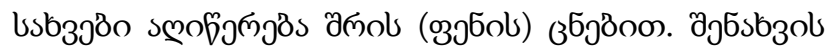

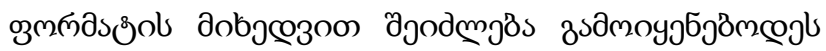

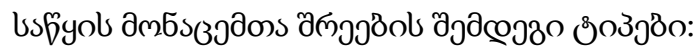

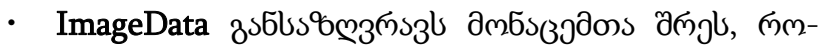

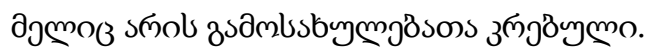

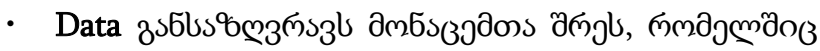

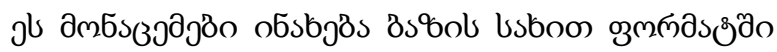
leveldb s5 lmdb.

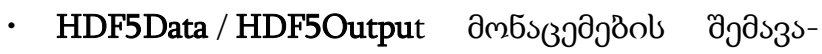

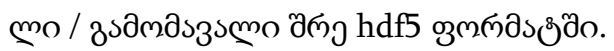

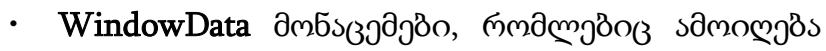

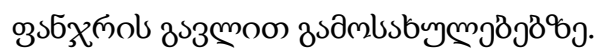

- เbаз.

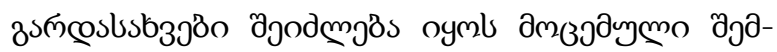

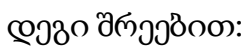

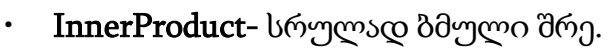

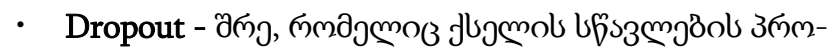
в з

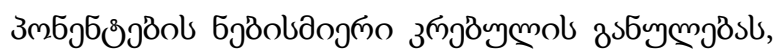

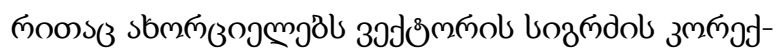
తింngBsl.

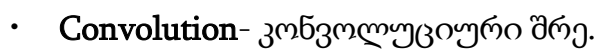

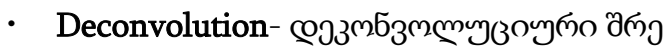

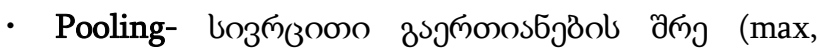
average, stochastic)

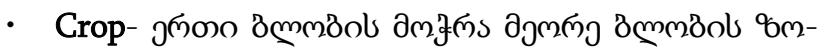

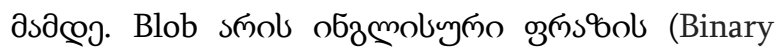

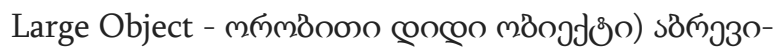

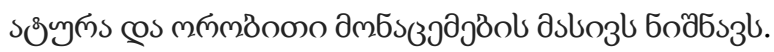




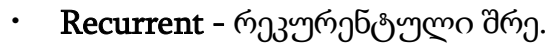

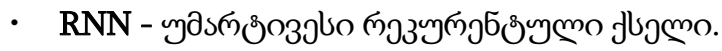

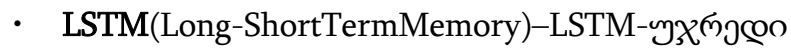

- LRN (Local Response Normalization) - mмззммуп

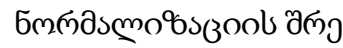

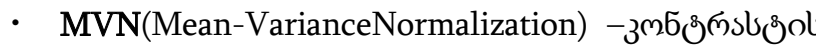

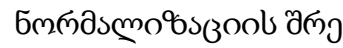

- BatchNorm(BatchNormalization)

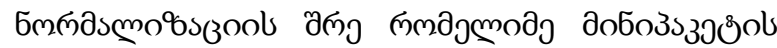
dobjœ̋ంon (minibatch).

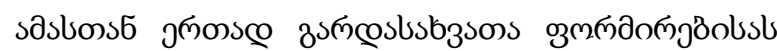

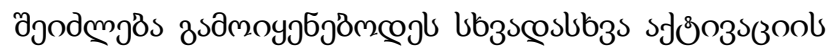
oỹficos.

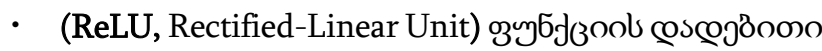

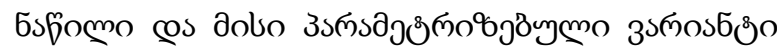
(PReLU).

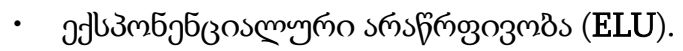

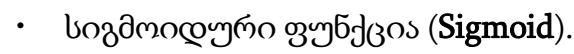

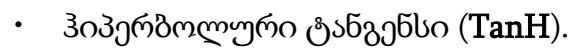

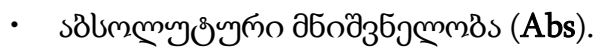

- sbsmolbjäs, sбy bsmobbðon syzs6s (Power).

- ммдзпоподо (Log).

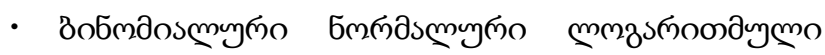

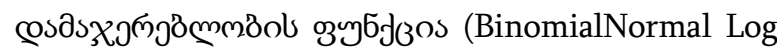
Likelihood,BNLL).

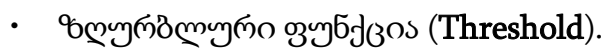

- 6s5s (33mg8s (Bias).

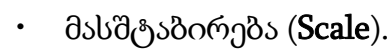

блопмды

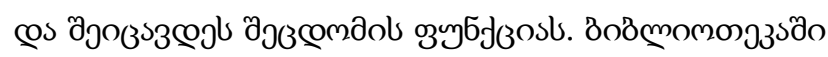

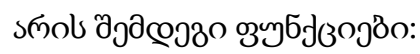

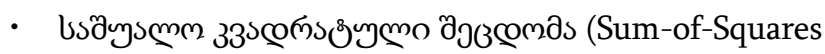
/ Euclidean Loss Layer,EuclideanLoss).

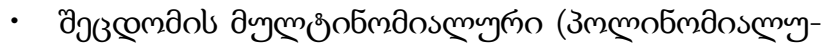

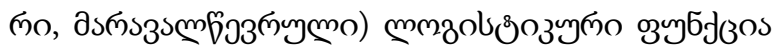
(Multinomial LogisticLoss, MultinomialLogisticLoss).

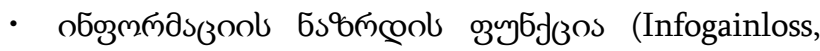
InfogainLoss).

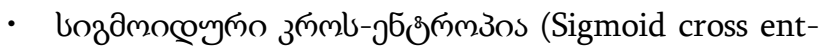
ropy loss, SigmoidCrossEntropyLoss).

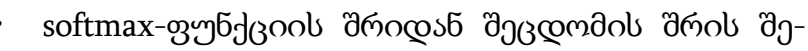

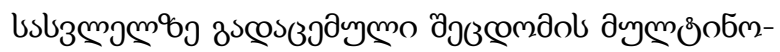

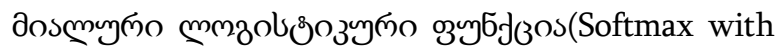
Loss,SoftmaxWithLoss).

- bb3s.

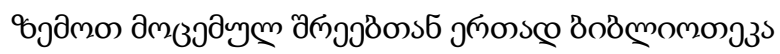

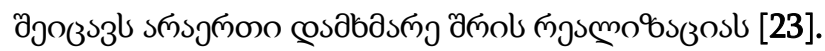

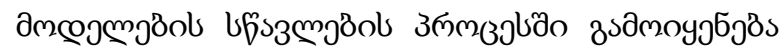

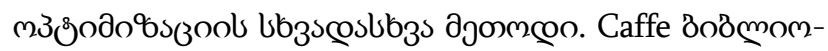

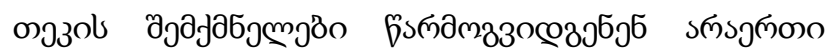

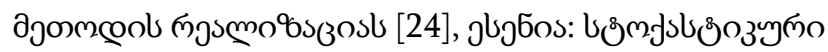

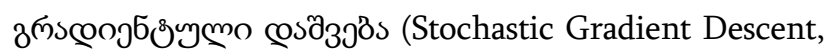

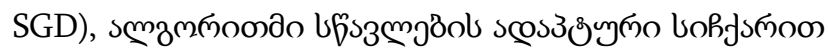
(Adaptive Gradient Learning Rate Algorithm, AdaGrad),

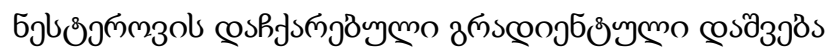
(Nesterov's Accelerated Gradient Descent, NAG),

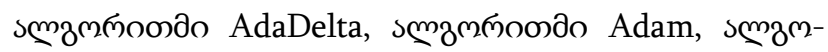

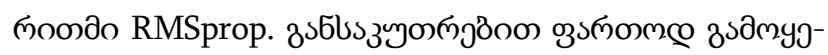

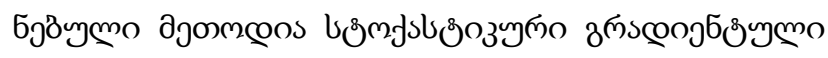
$\cos \partial_{3 j} \mathrm{j}$ s.

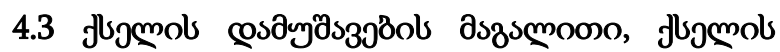

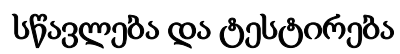

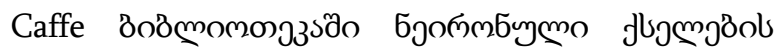

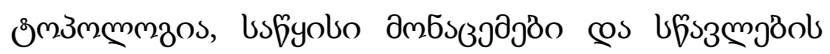

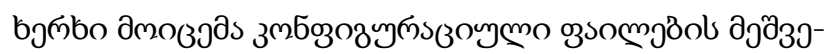

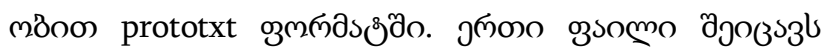




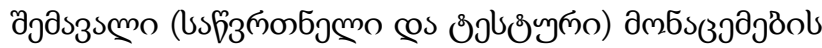

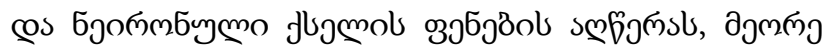

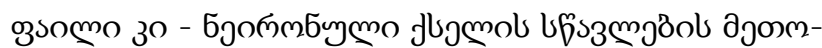

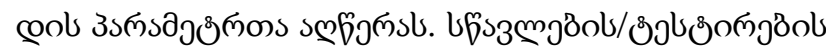

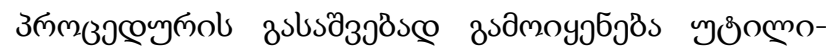

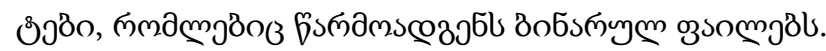

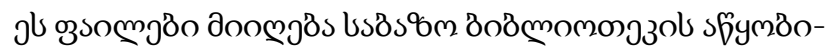

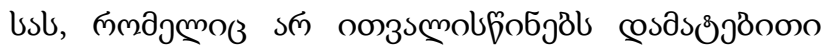

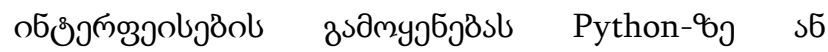
MATLAB-

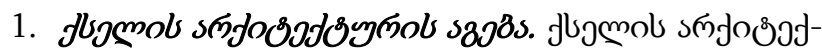

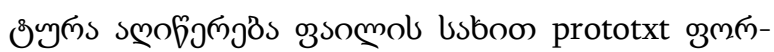

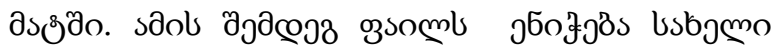

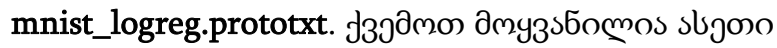

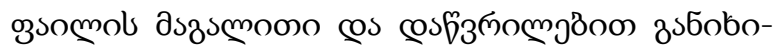

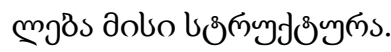

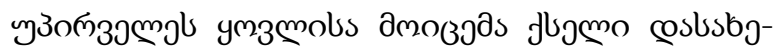
mgßs:

name:"LogisticRegression"

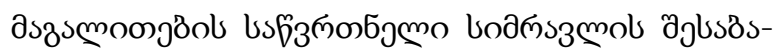

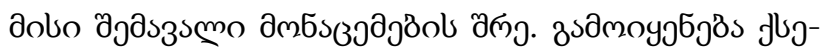

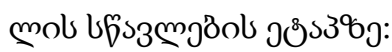

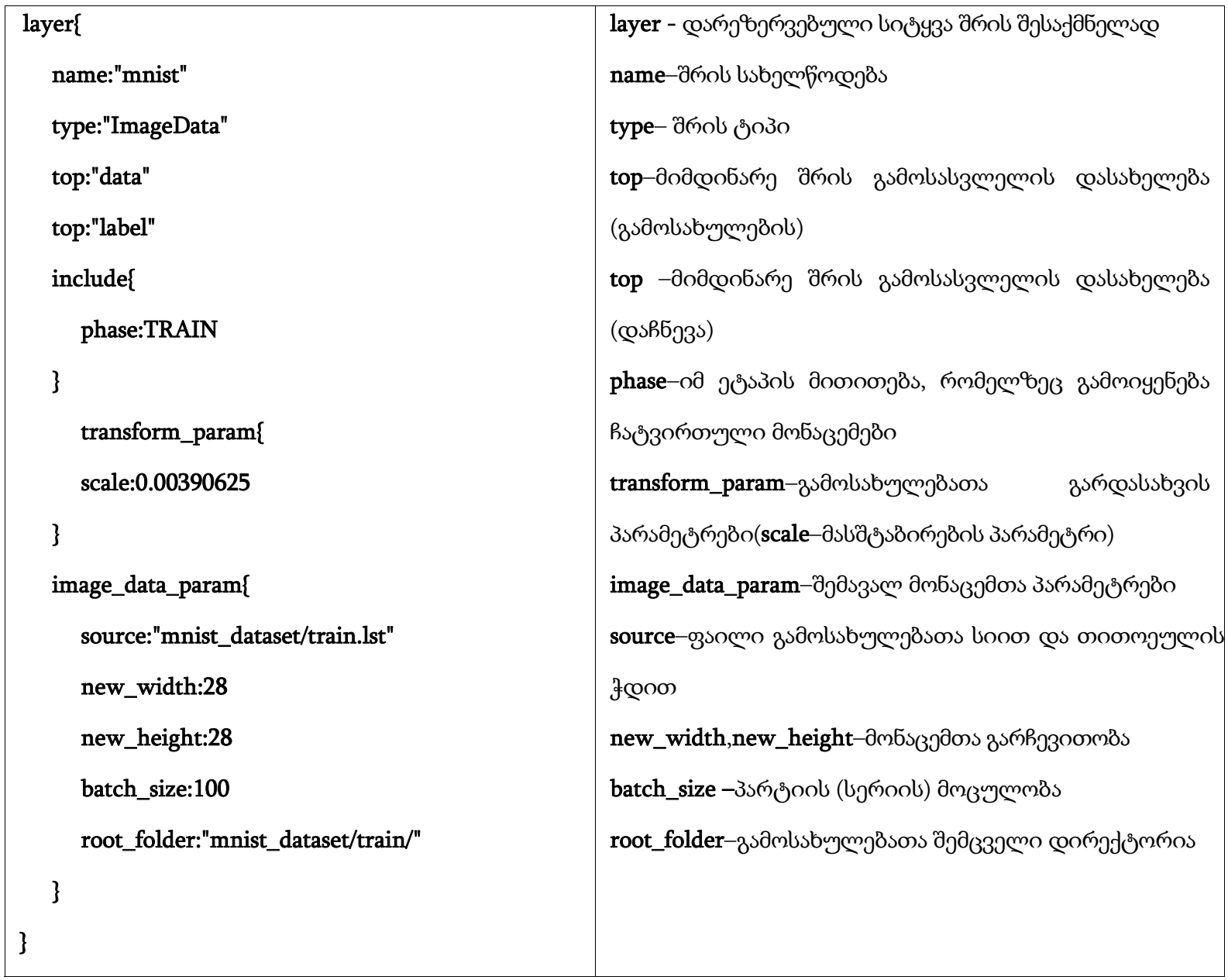




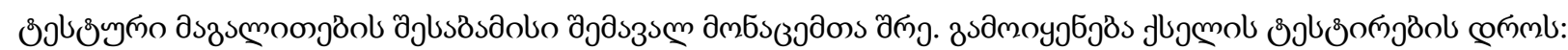

\begin{tabular}{|c|c|}
\hline $\begin{array}{l}\text { layer\{ } \\
\text { name:"mnist" } \\
\text { type:"ImageData" } \\
\text { top:"data" } \\
\text { top:"label" } \\
\text { include\{ } \\
\text { phase:TEST } \\
\text { \} } \\
\text { transform_param\{ } \\
\text { scale:0.00390625 } \\
\text { \} } \\
\text { image_data_param\{ } \\
\text { source:"mnist_dataset/t10k.lst" } \\
\text { new_width:28 } \\
\text { new_height:28 } \\
\text { batch_size:100 } \\
\text { root_folder:"mnist_dataset/t10k/" } \\
\text { \} }\end{array}$ & 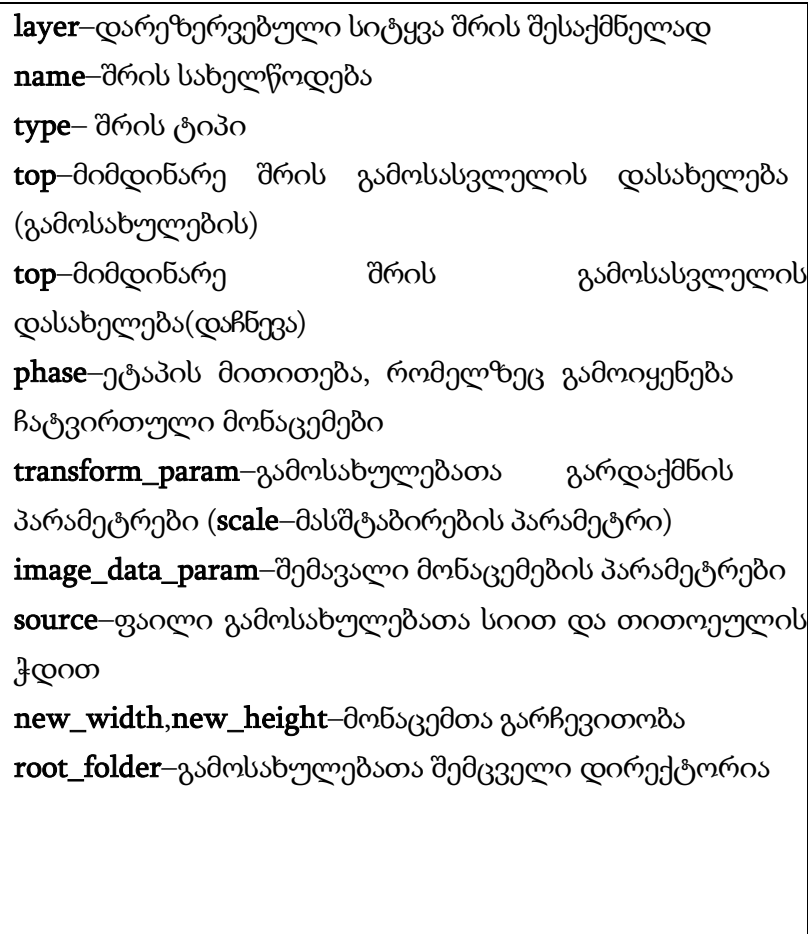 \\
\hline
\end{tabular}

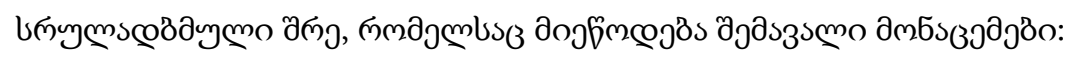

\begin{tabular}{|c|c|}
\hline $\begin{array}{l}\text { layer\{ } \\
\text { name:"ip" } \\
\text { type:"InnerProduct" } \\
\text { bottom:"data" } \\
\text { top:"ip" } \\
\text { inner_product_param\{ } \\
\quad \text { num_output:10 } \\
\text { \} }\end{array}$ & 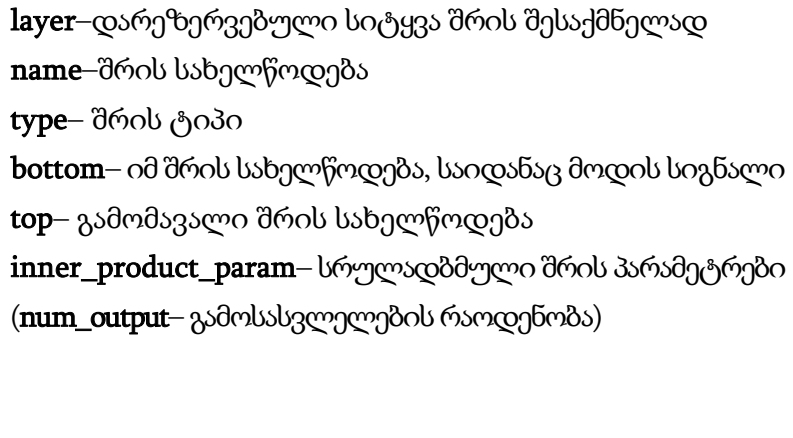 \\
\hline
\end{tabular}

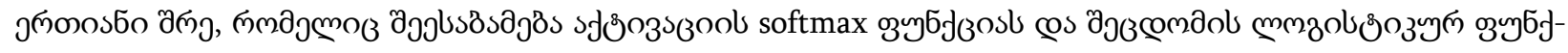

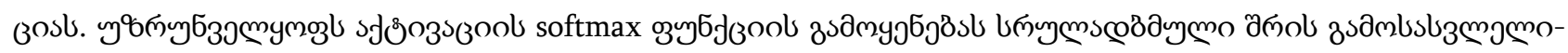

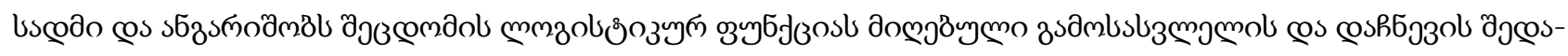

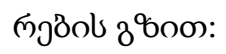

\begin{tabular}{|c|c|}
\hline $\begin{array}{l}\text { layer\{ } \\
\text { name:"loss" } \\
\text { type:"SoftmaxWithLoss" } \\
\text { bottom:"ip" } \\
\text { bottom:"label" } \\
\text { top:"loss" }\end{array}$ & 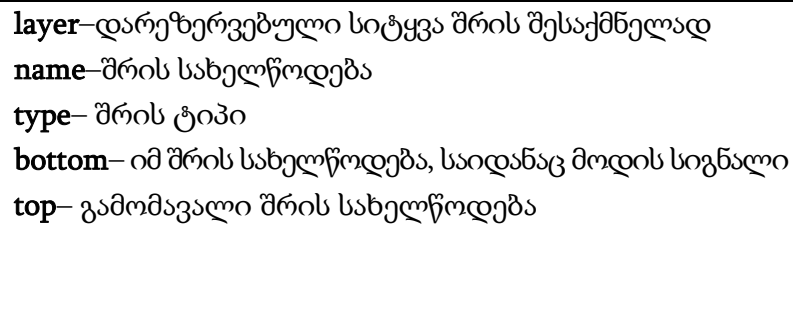 \\
\hline
\end{tabular}


з

\begin{tabular}{|c|c|}
\hline $\begin{array}{l}\text { layer\{ } \\
\text { name:"accuracy" } \\
\text { type:"Accuracy" } \\
\text { bottom:"ip" } \\
\text { bottom:"label" } \\
\text { top:"accuracy" } \\
\text { include\{ } \\
\text { phase:TEST } \\
\text { \} }\end{array}$ & 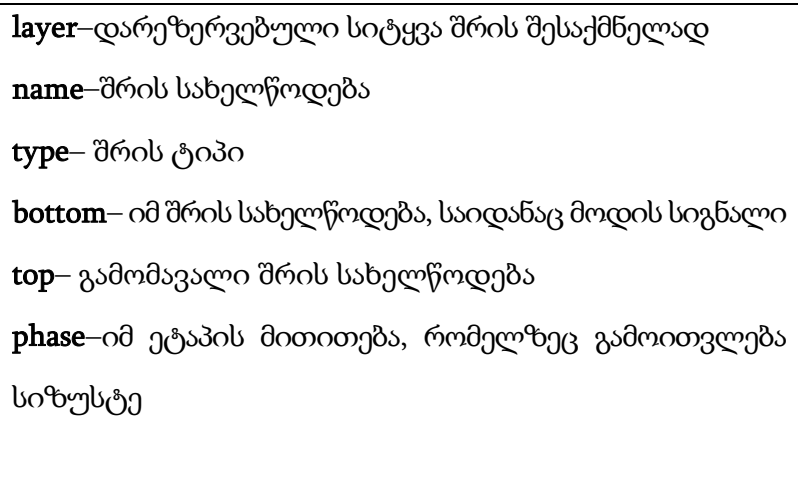 \\
\hline
\end{tabular}

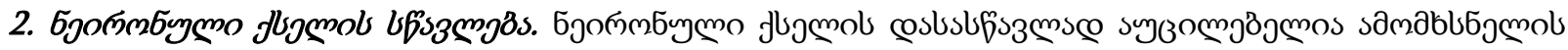

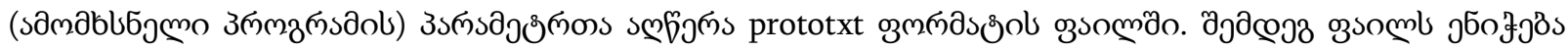

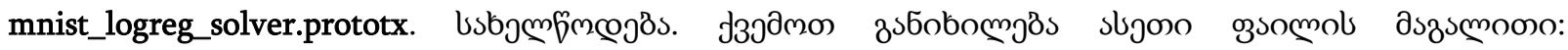

\begin{tabular}{|c|c|}
\hline $\begin{array}{l}\text { net:"mnist_logreg.prototxt" } \\
\text { test_iter:100 } \\
\text { test_interval:500 } \\
\text { base_lr:0.01 } \\
\text { momentum:0.9 } \\
\text { weight_decay:0.0005 } \\
\text { lr_policy:"inv" } \\
\text { gamma:0.0001 } \\
\text { power:0.75 } \\
\text { display:100 } \\
\text { max_iter:10000 } \\
\text { snapshot:5000 } \\
\text { snapshot_prefix:"mnist_logreg" } \\
\text { solver_mode:CPU }\end{array}$ & 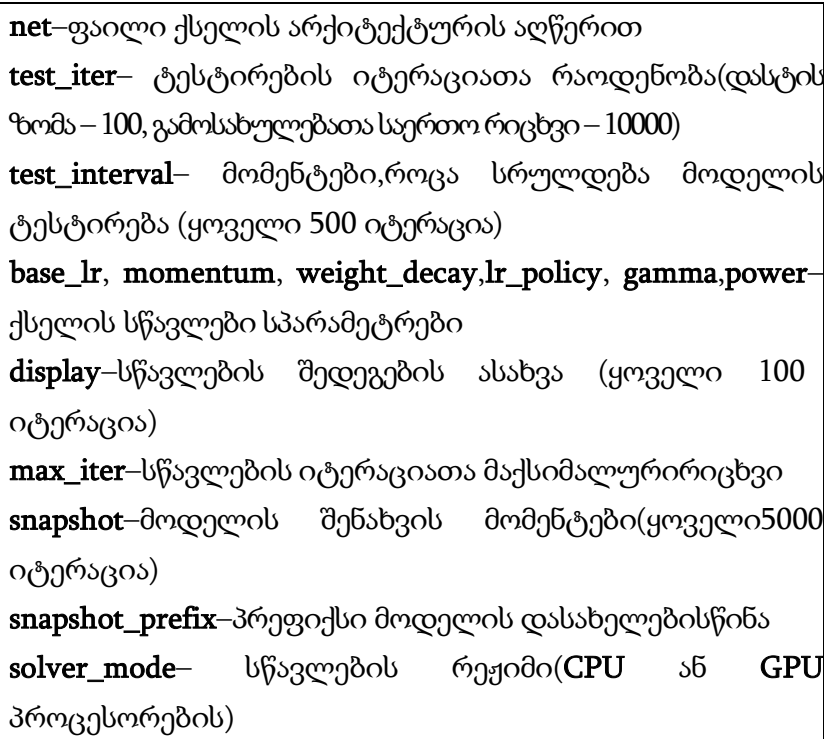 \\
\hline
\end{tabular}

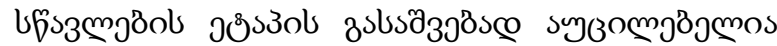

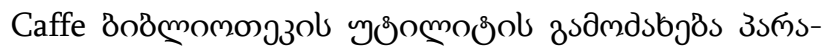

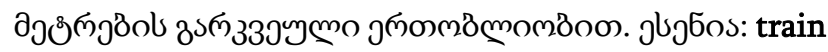

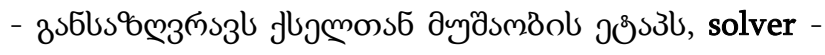

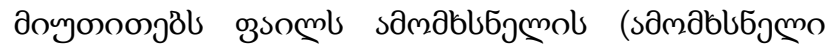

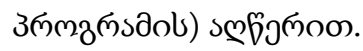

\$CAFFE_ROOT/build/tools/caffetrain--solver= mnist_logreg_solver.prototxt\$@

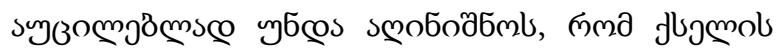

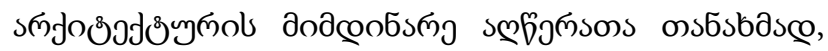

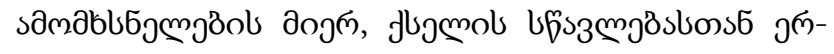




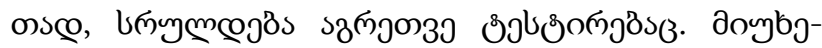

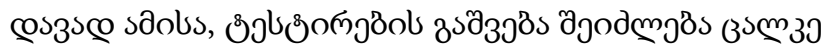

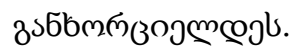

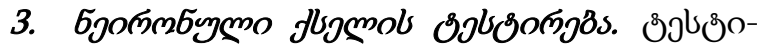

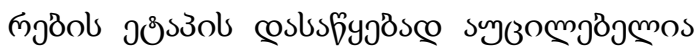

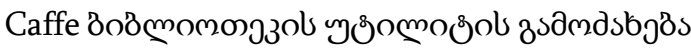

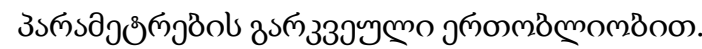

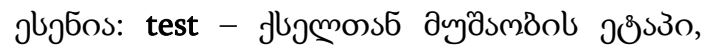

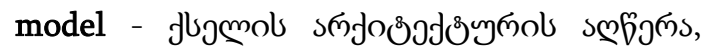

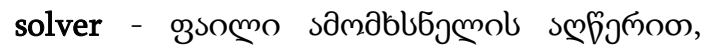

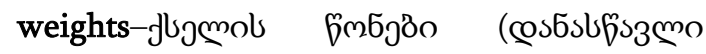
дмœумо)

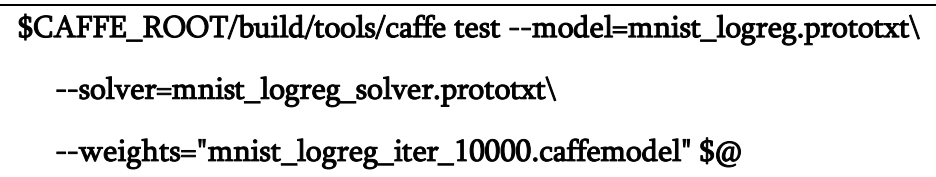

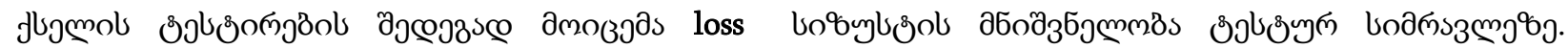

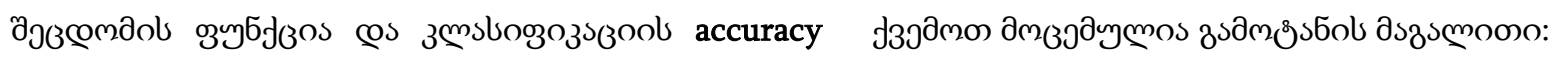

$\begin{array}{ll}\text { I1016 16:13:53.621152 } & 6448 \text { caffe.cpp:330] accuracy }=0.9062 \\ \text { I1016 16:13:53.621218 } & 6448 \text { caffe.cpp:330] loss }=0.343764\left({ }^{*} 1=0.343764 \text { loss }\right)\end{array}$

\section{5 довмомолјз Torch}

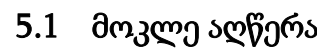

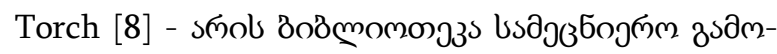

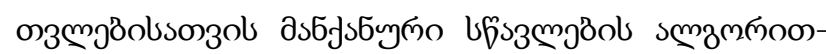

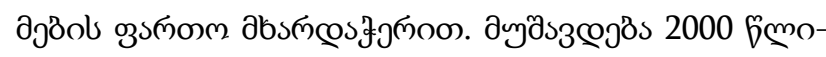
cos6 objo bsa Research Institute, New York University @os NEC Labo-

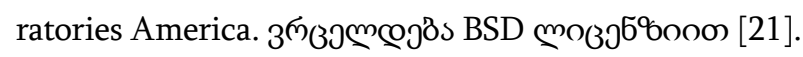

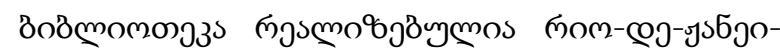

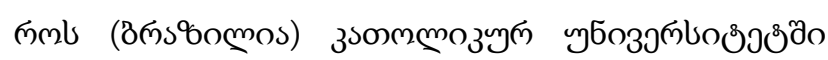

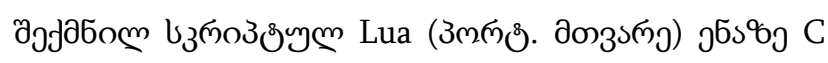
j5obs as CUDA (Compute Unified Device Architec-

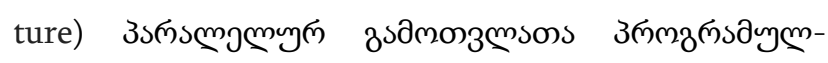

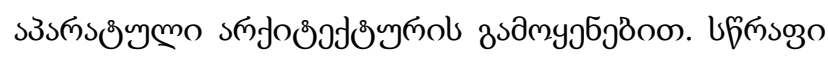

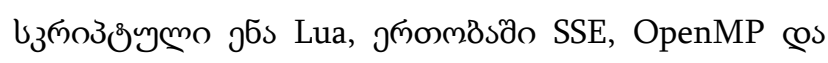

\section{ISSN 1512-0996}

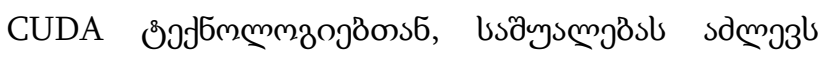

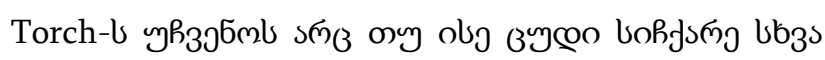

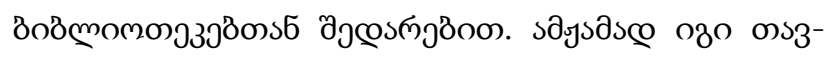

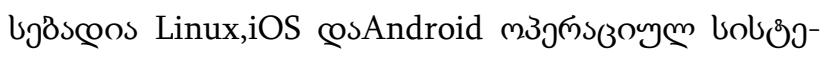

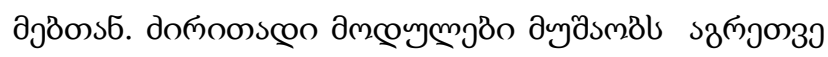

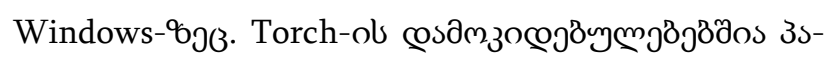

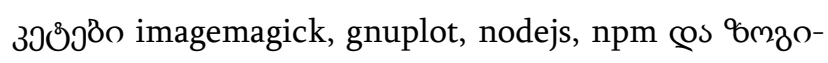
gตno bb3s.

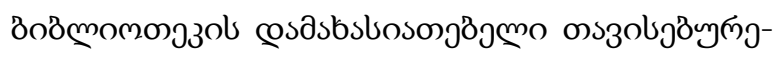

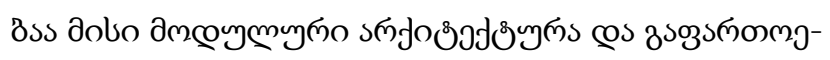

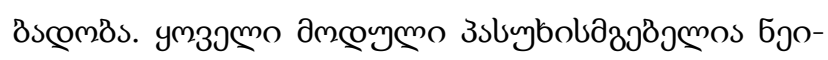

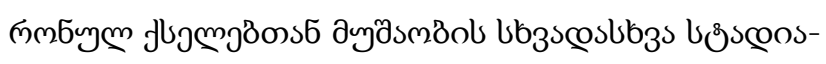

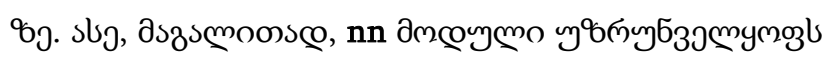

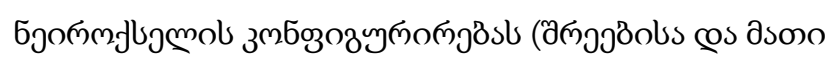

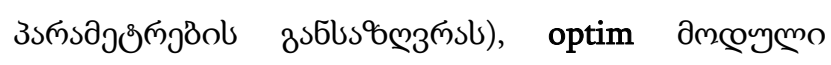




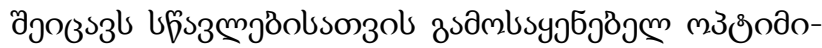

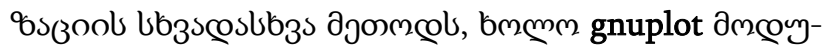

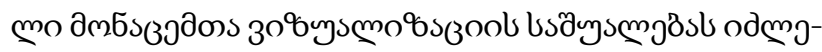

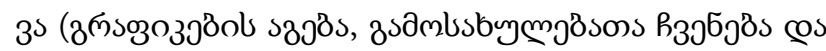

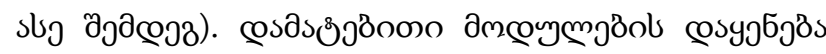

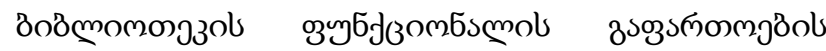
usäysmgäsls oдmэзs [21].

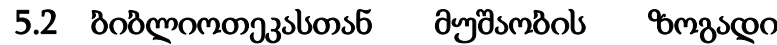

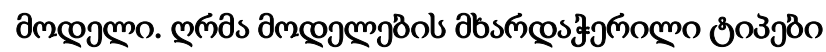

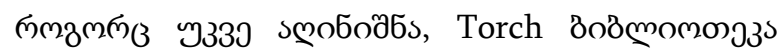

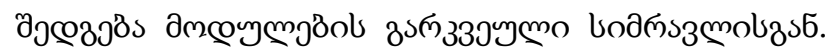

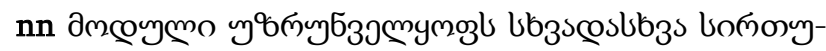

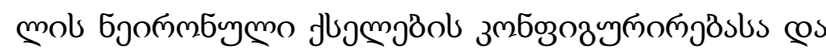

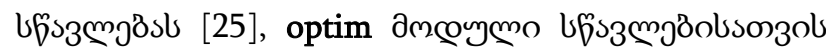

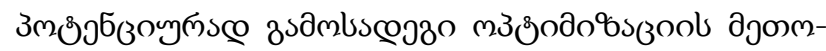

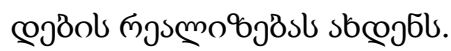

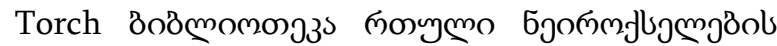

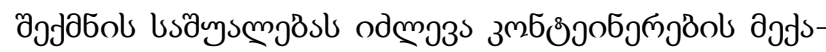

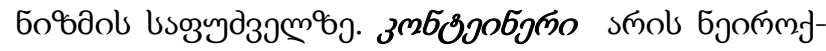

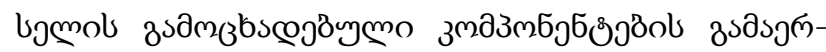
поsб

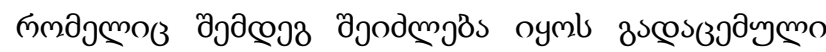

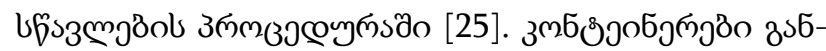

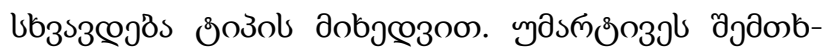

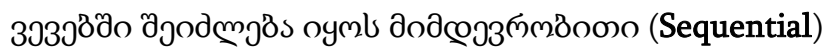

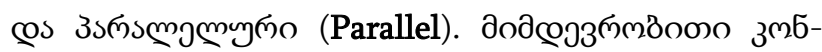

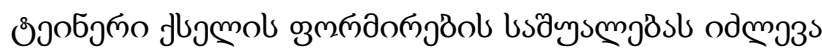

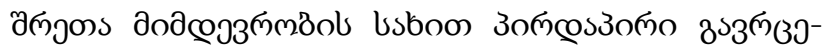

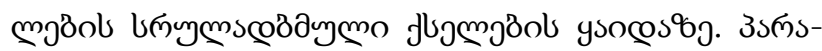

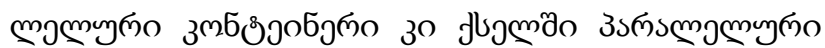

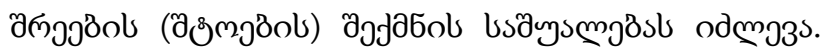

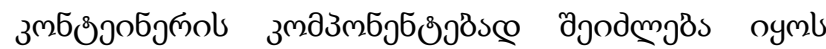

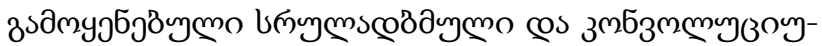

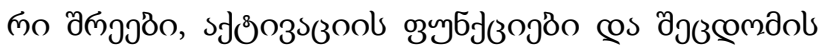

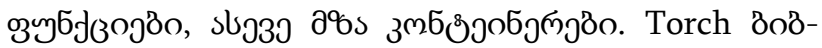

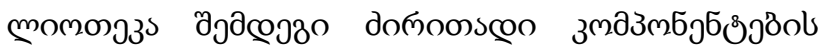

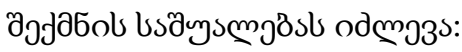

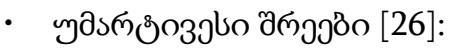

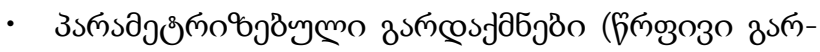
œs

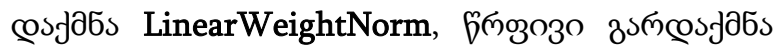

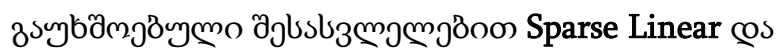
ubss);

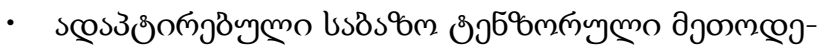

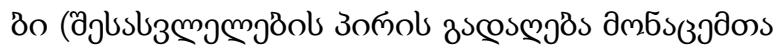

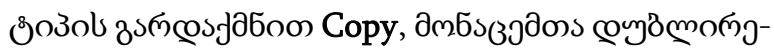

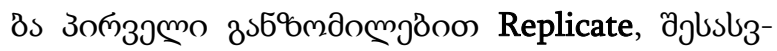

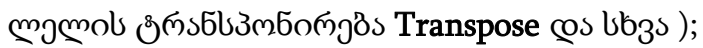

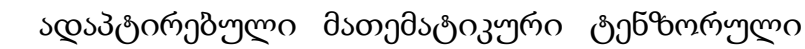

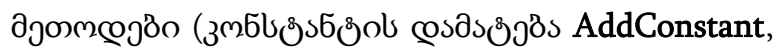

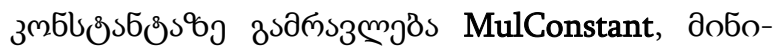

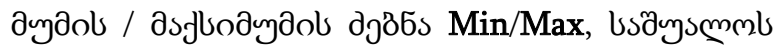

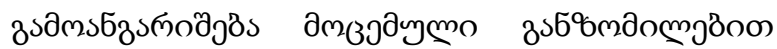

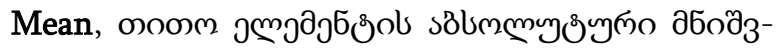

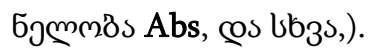

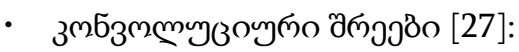

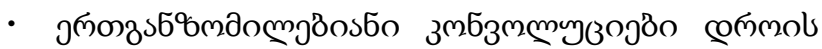

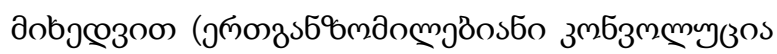

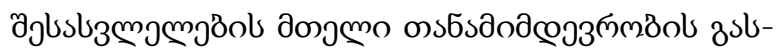

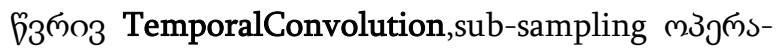

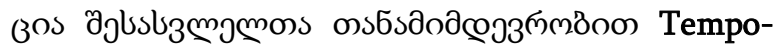

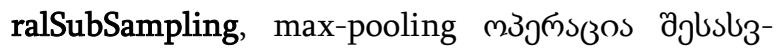

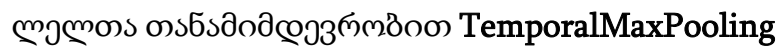
cos tb3s); 


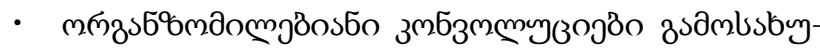

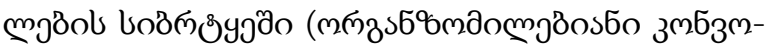
myzos zsambsbymgis\%g SpatialConvolution,

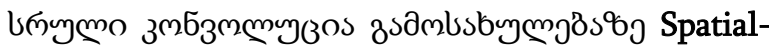

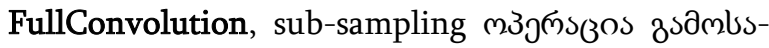
buym $383 \%$ g SpatialSubSampling, max-pooling m3ว-

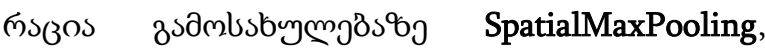

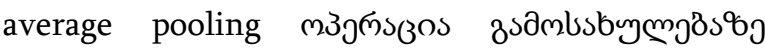
SpatialAveragePooling cos bb3s);

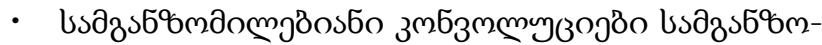

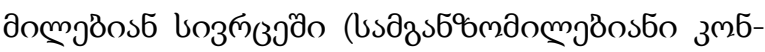

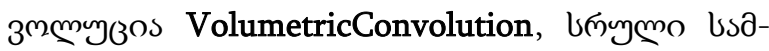

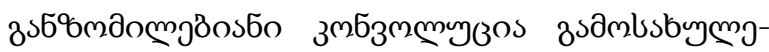
Bsons додеэз

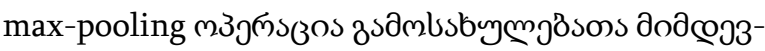
๓mBs\%g VolumetricMaxPooling, average pooling

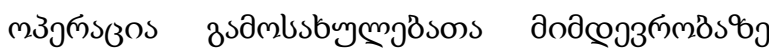
VolumetricAveragePooling (os bb3s).

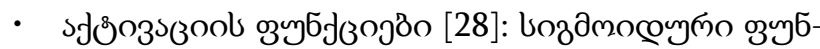

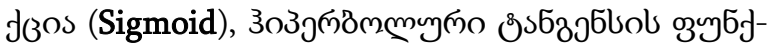
(3Os (Tanh), soft-max ojyбf(3Os (SoftMax), soft-min

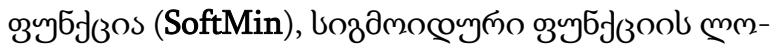

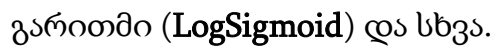

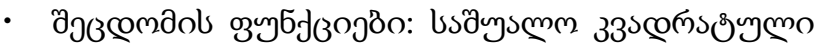

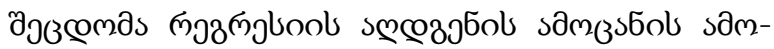

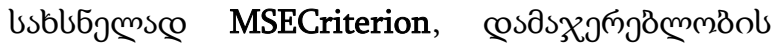

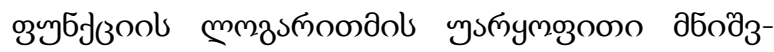

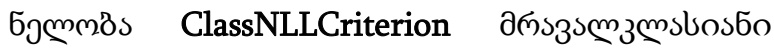
zensbogrzszool samzistobsonzol.

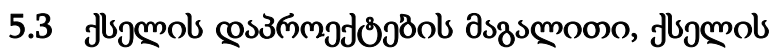

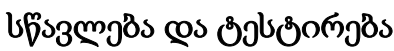

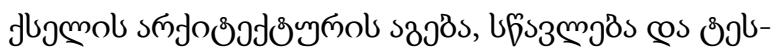

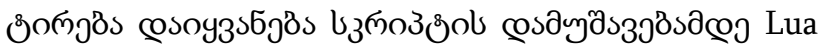

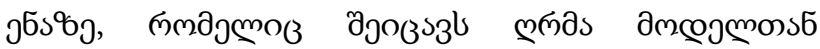

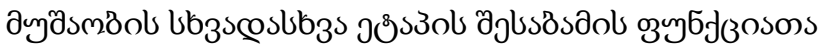
उ

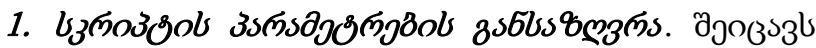

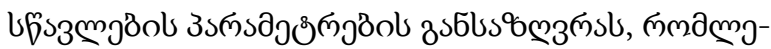

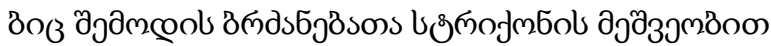

\footnotetext{
local opt $=$ lapp[[

-s,--save(default "logs")

-f,--full

-p,--plot

-r,--learningRate(default 0.05)

-b,--batchSize(default 0)

-m,--momentum(default 0)

--coefL1(default 0)

--coefL2(default 0)

-t,--threads(default 4)

]]
}

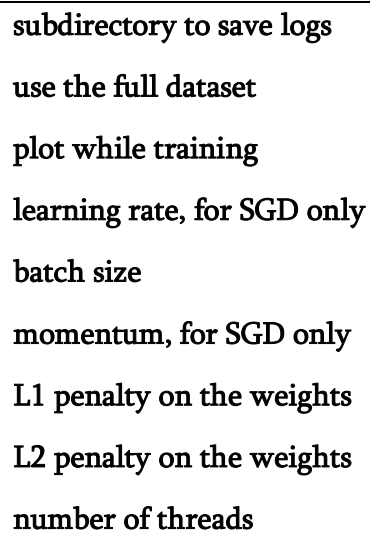

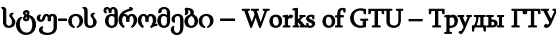




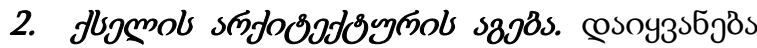

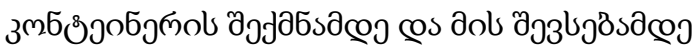
ฆ๓ววठักั.

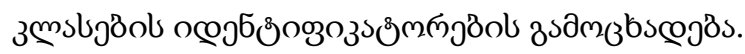

classes= $=\{1$ ', '2','3','4','5','6', '7', '8','9','10'\}

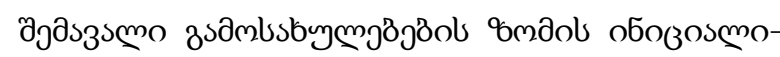
gososos.

geometry $=\{32,32\}$

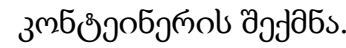

model $=$ nn.Sequential ()

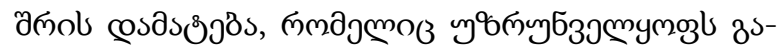

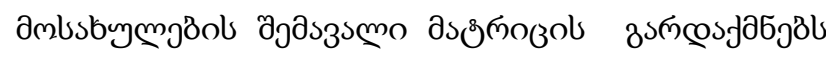

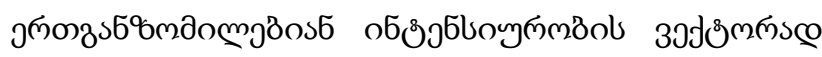

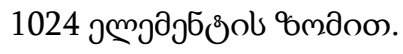

model:add(nn.Reshape(1024))

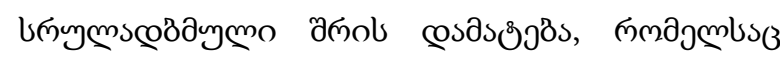

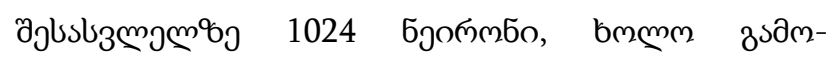

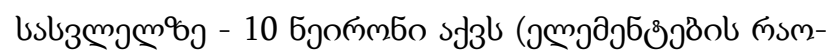
๔jб

model:add(nn.Linear(1024,\#classes))

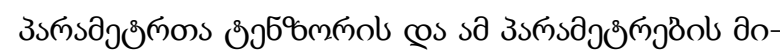

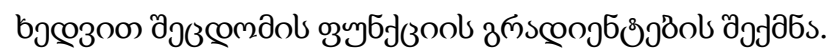

parameters, gradParameters $=$ model:getParameters ()

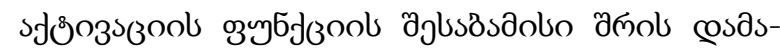
उิ38s.

model:add(nn.LogSoftMax())

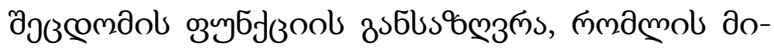

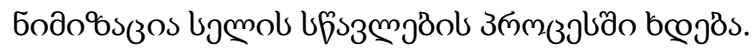
model:add(nn.Linear(1024,\#classes))

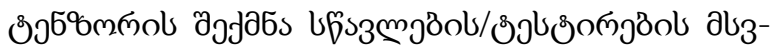

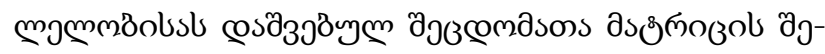
usbsbsse.

confusion $=$ optim. ConfusionMatrix (classes)

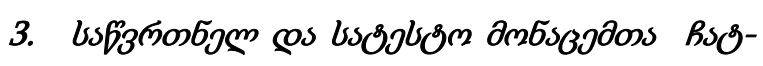

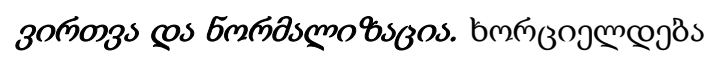

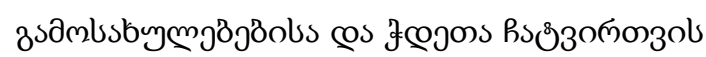

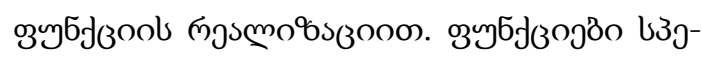

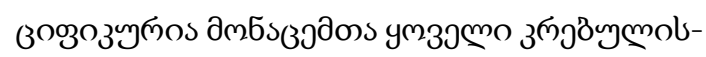

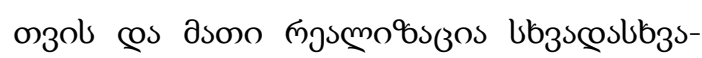

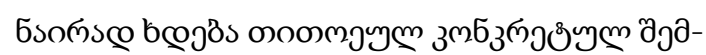
ob333sð̃o.

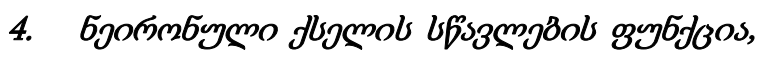

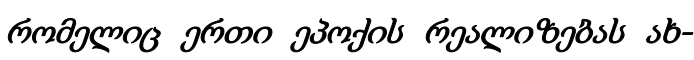

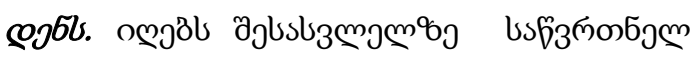

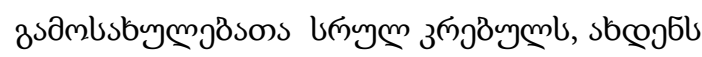

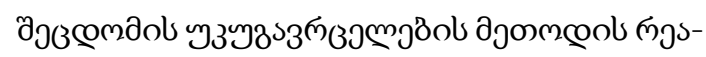

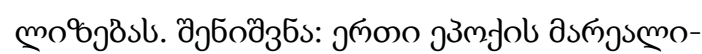

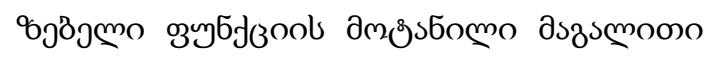

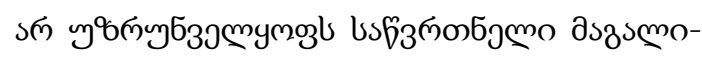

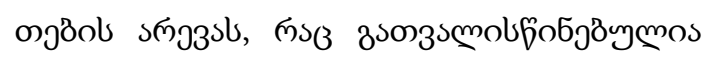

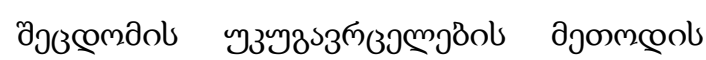

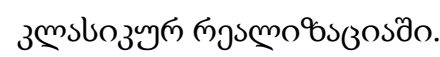

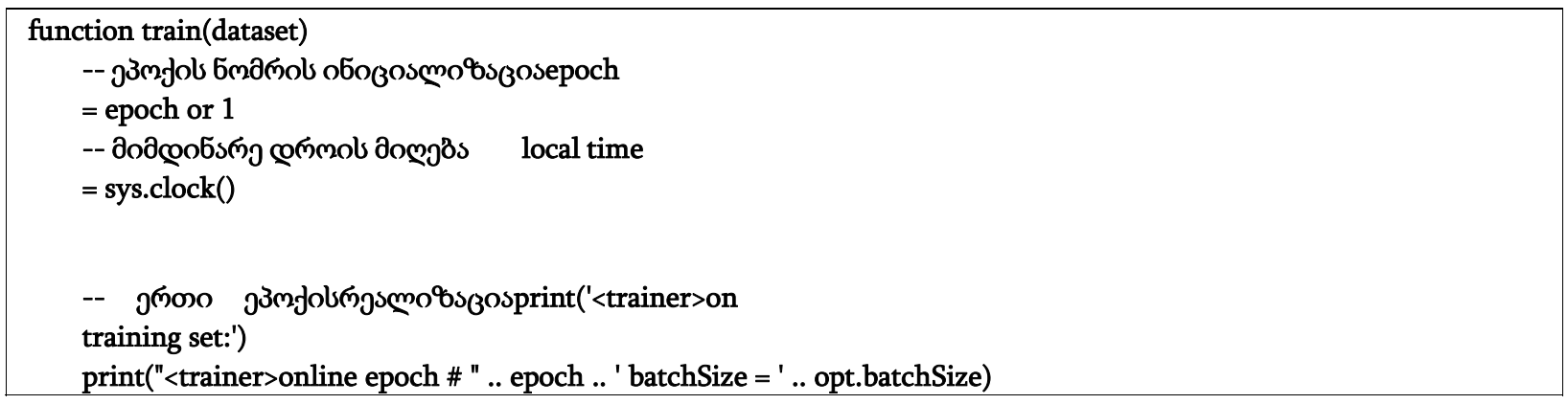




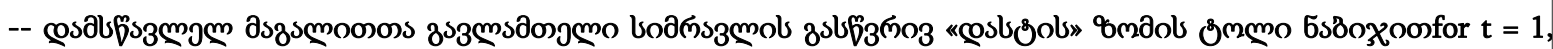
dataset:size(), opt.batchSize do

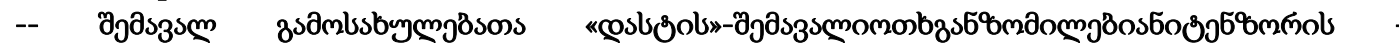

ӘəЈӘбslocalinputs = torch.Tensor(opt.batchSize,1,geometry[1],geometry[2])

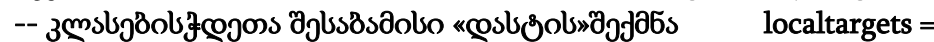

torch.Tensor(opt.batchSize)

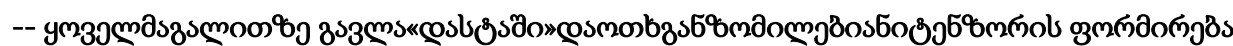

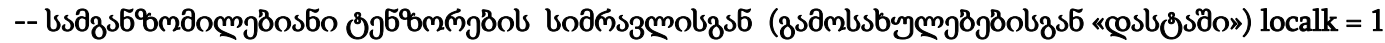

for $\mathrm{i}=\mathrm{t}$, math.min(t+opt.batchSize-1, dataset:size ()$)$ do

-- амково aszumoools

дощృठ̊slocalsample $=$ dataset[i]

-- zsambsbuymjas

local input $=$ sample[1]:clone ()

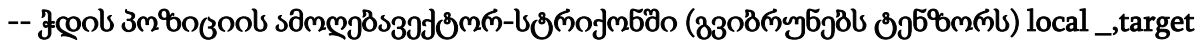

= sample[2]:clone ()$: \max (1)$

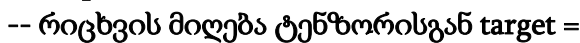

target:squeeze() inputs[k] = input

targets $[\mathrm{k}]=$ target $\mathrm{k}=\mathrm{k}+1$

end

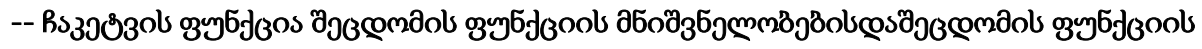

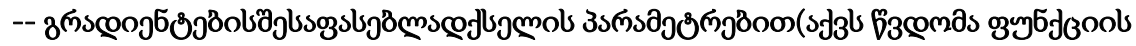

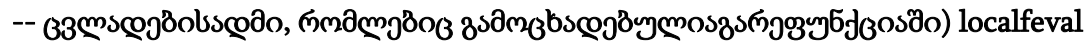

$=$ function $(\mathrm{x})$

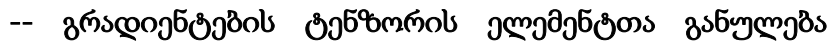

gradParameters:zero()

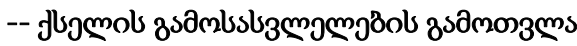

local outputs = model:forward(inputs)

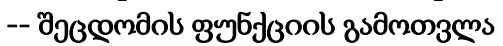

local $\mathrm{f}=$ criterion:forward(outputs, targets)

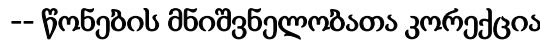

local df_do = criterion:backward(outputs, targets)

model:backward(inputs, df_do)

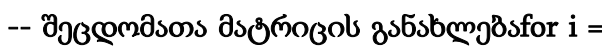

1, opt.batchSize do

confusion:add(outputs[i], targets[i])

end

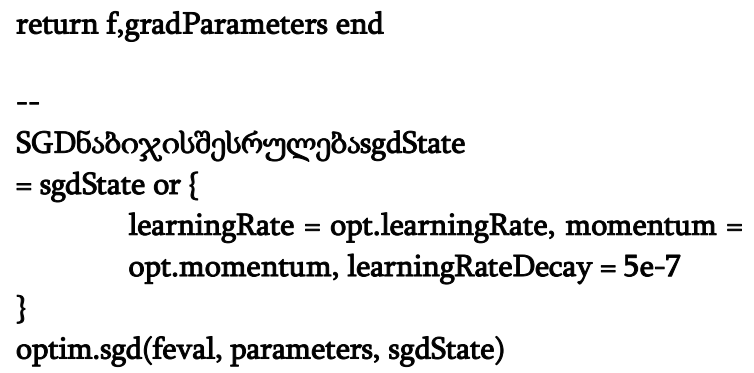




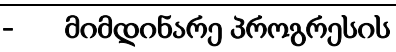

slsbb3saxlua.progress(t, dataset:size())

end

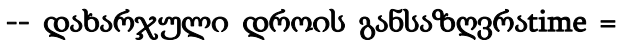

sys.clock () - time

time $=$ time $/$ dataset:size()

print $("<$ trainer>time to learn 1 sample $=$ " .. (time*1000) .. 'ms')

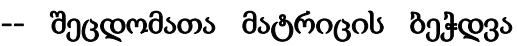

print(confusion)

trainLogger:add $\{[$ '\% mean class accuracy (train set)']=confusion.totalValid * 100$\}$ confusion:zero()

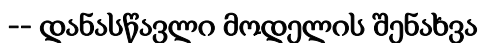

local filename = paths.concat(opt.save, 'mnist.net') os.execute('mkdir -p ' ..

sys.dirname(filename))

if paths.filep(filename) then

os.execute('mv' .. filename .. ' ' .. filename .. '.old') end

print('<trainer>saving network to '..filename) torch.save(filename,

model)

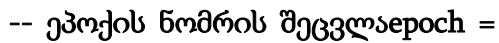

epoch +1

end

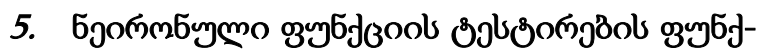

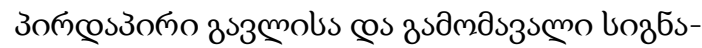

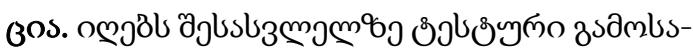

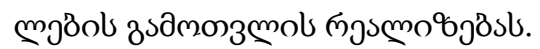

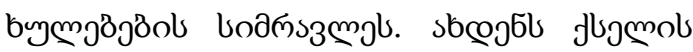

functiontest(dataset)

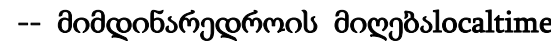

$=$ sys.clock ()

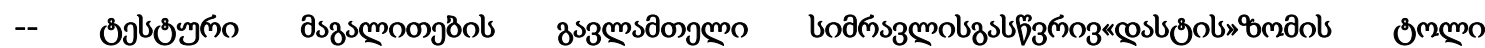
6sð̊oxoonprint('<trainer>ontestingSet:')

for $t=1$, dataset:size(), opt.batchSize do

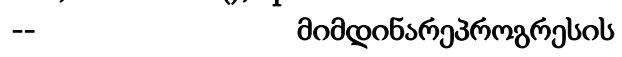

sbsb3sxlua.progress(t, dataset:size())

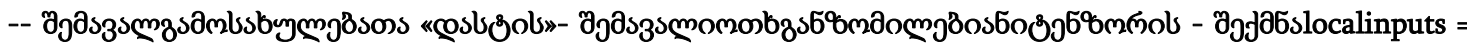
torch.Tensor(opt.batchSize,1,geometry[1],geometry[2])

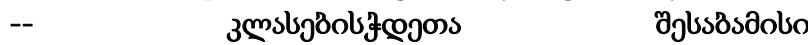

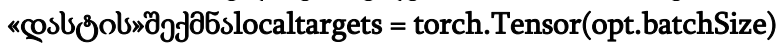

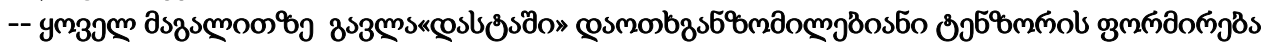

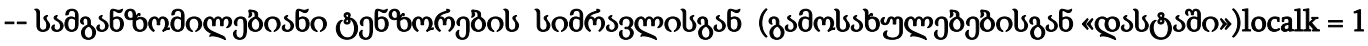

for $\mathrm{i}=\mathrm{t}$, math.min $(\mathrm{t}+$ opt.batchSize-1,dataset:size ()$)$ do

-- ампово aszumonos

aosjBslocalsample $=$ dataset $[\mathrm{i}]$

local input $=$ sample[1]:clone () 


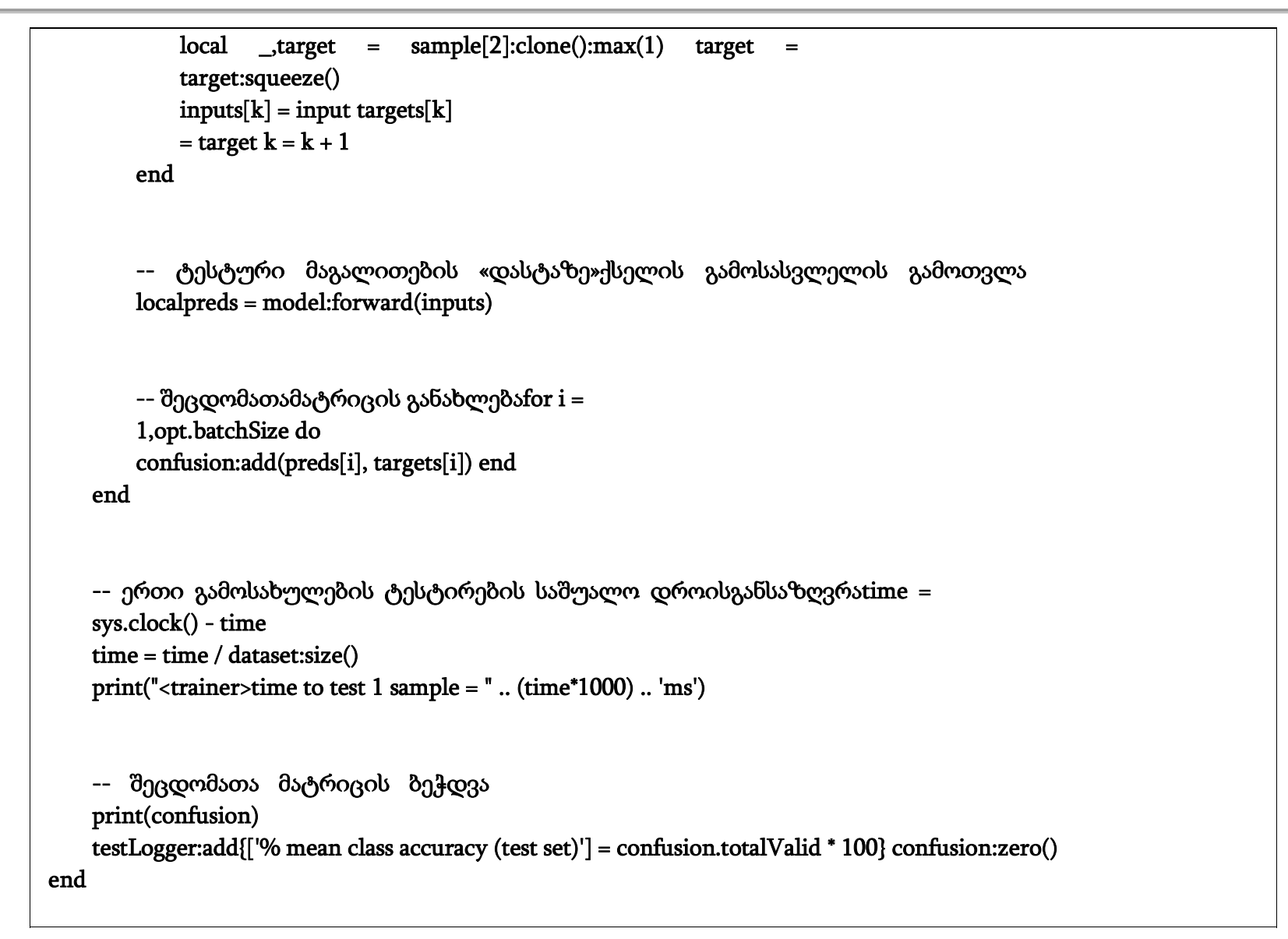

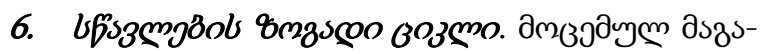

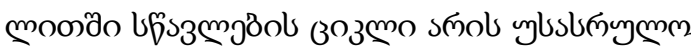

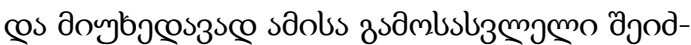

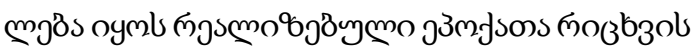

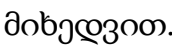

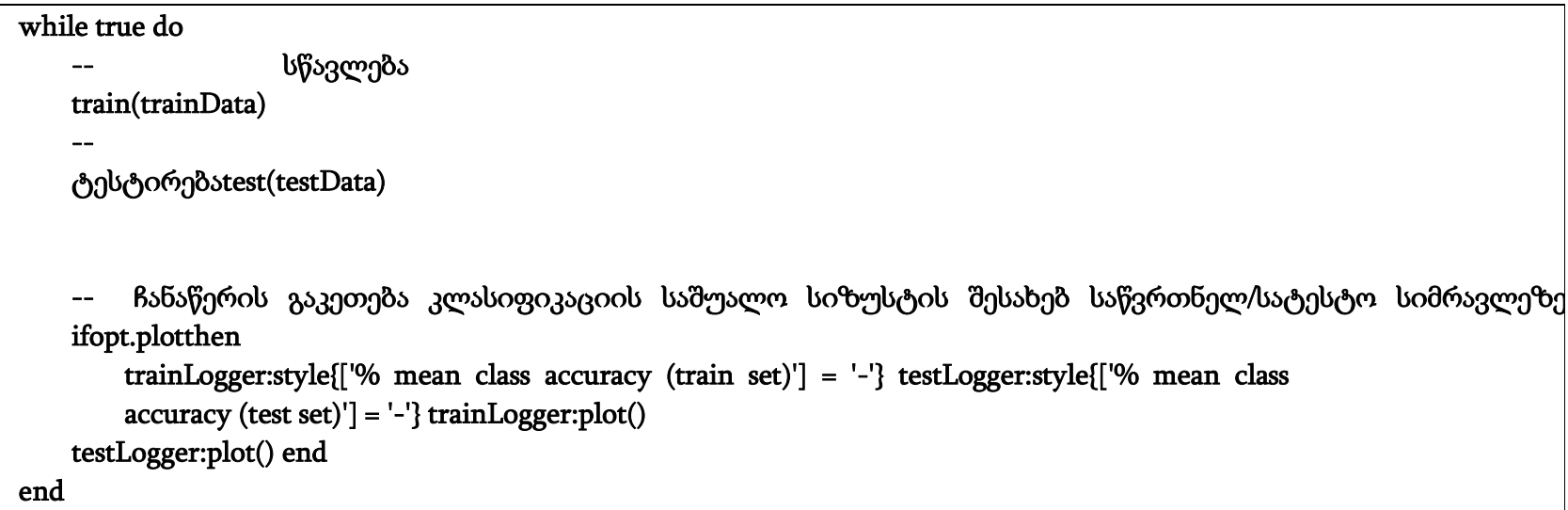




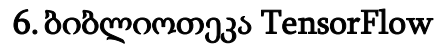

\section{1 дмзмл sмбэм}

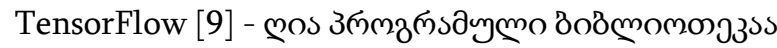

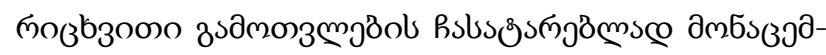

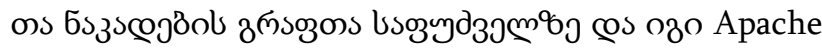

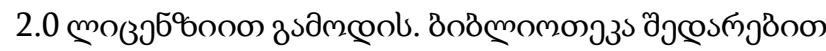

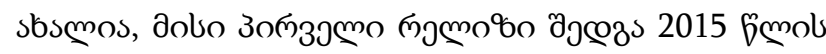

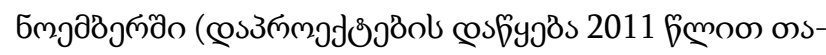

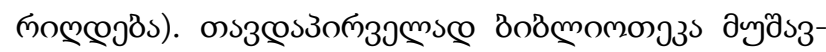

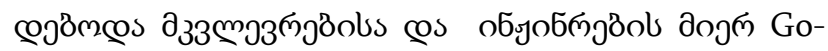

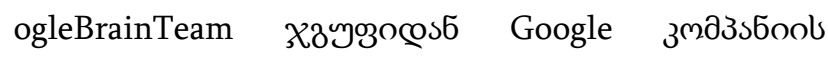
Machine Intelligence Research Organization

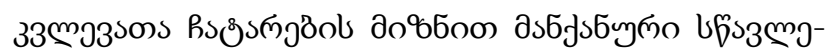

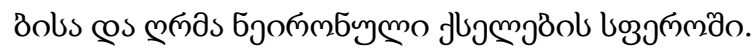

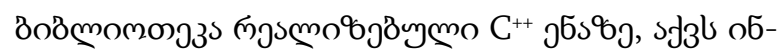

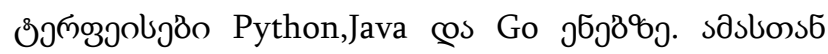

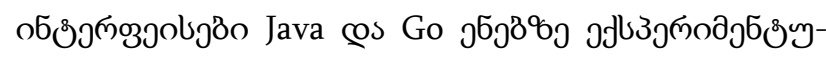

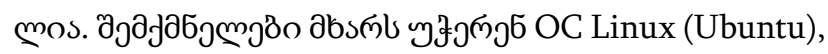

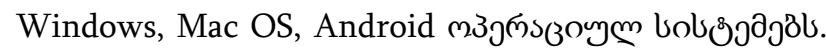

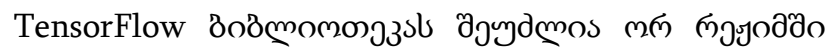

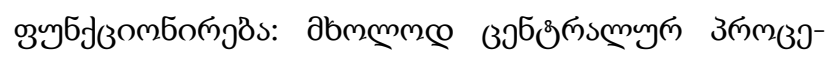

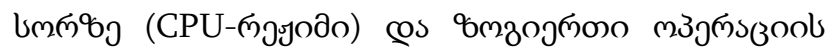

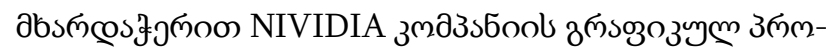

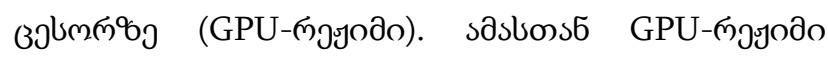

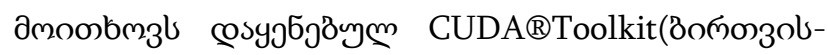

CUDA, Compute Unified Device Architecture) bsð̃ys-

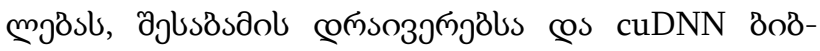
mомолзы [29].

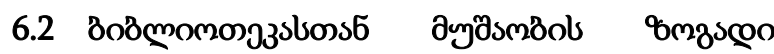

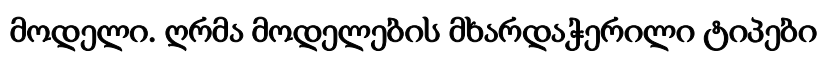

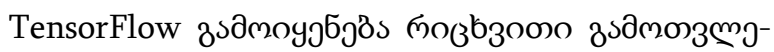

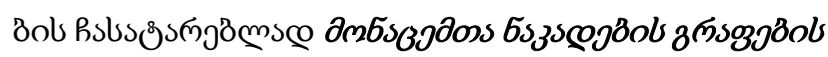

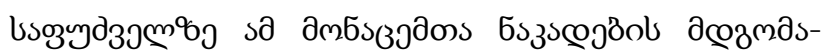

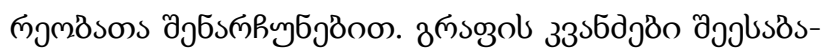

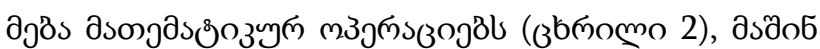

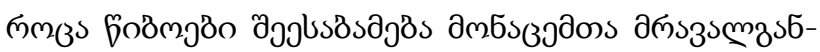

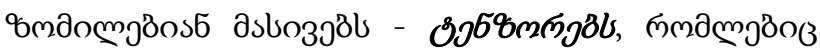

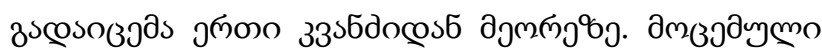

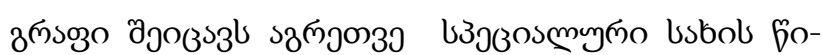

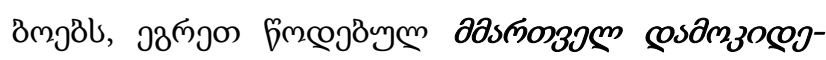

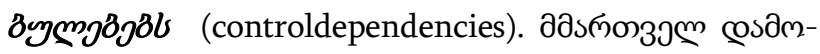

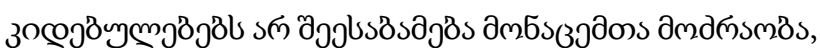

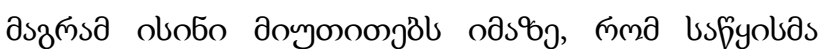

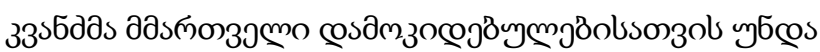

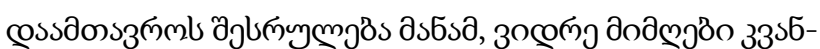

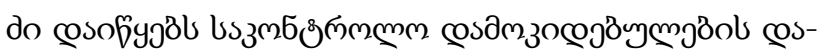

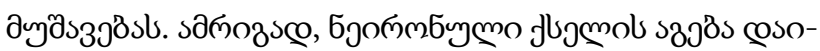

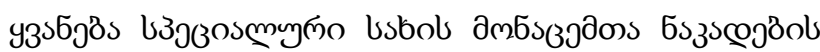

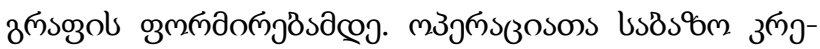
вээmol s๓ు

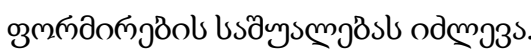




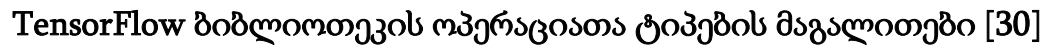

\begin{tabular}{|c|c|}
\hline 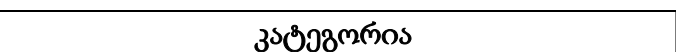 & 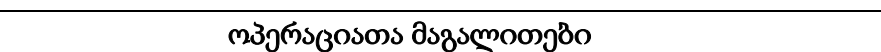 \\
\hline 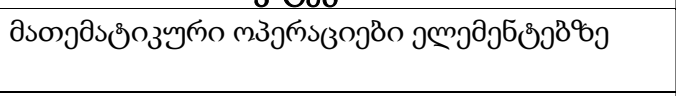 & 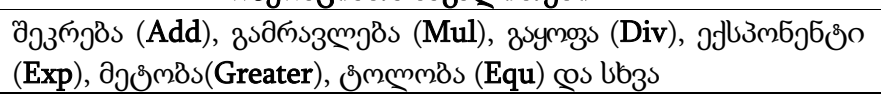 \\
\hline 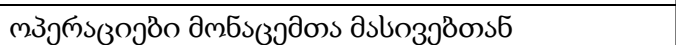 & z১sos \\
\hline 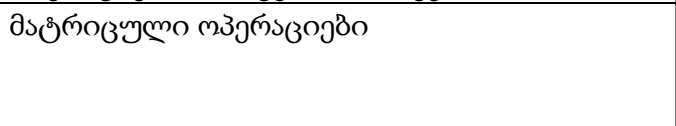 & 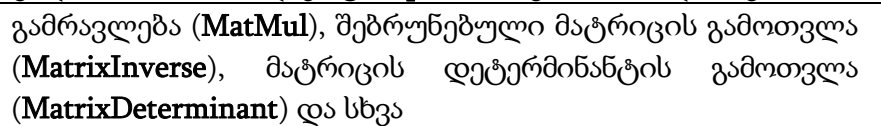 \\
\hline 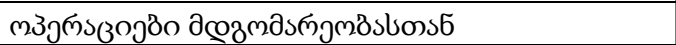 & 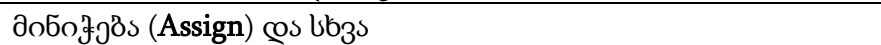 \\
\hline 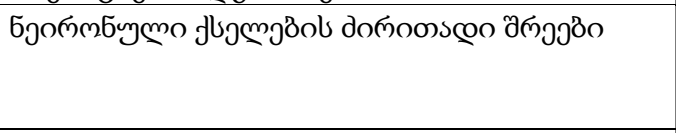 & 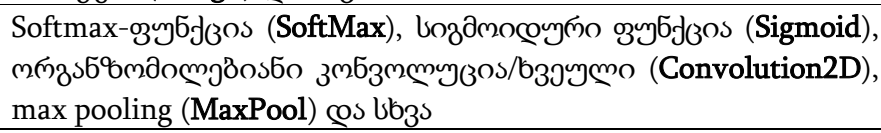 \\
\hline 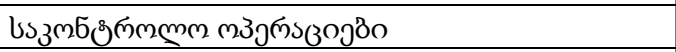 & 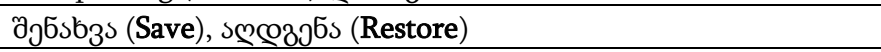 \\
\hline 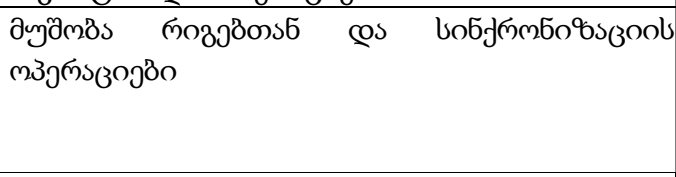 & 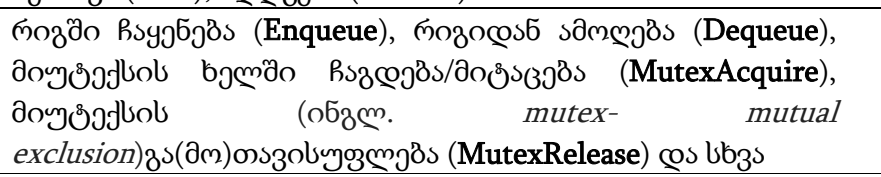 \\
\hline 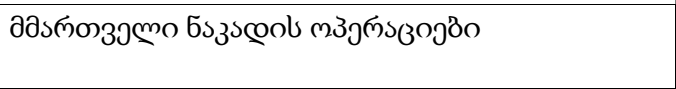 & 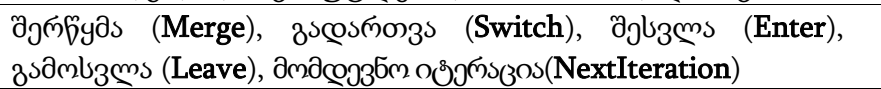 \\
\hline
\end{tabular}

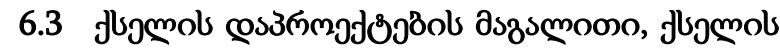

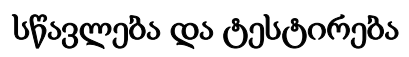

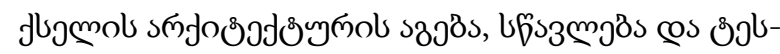

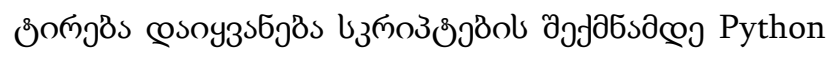

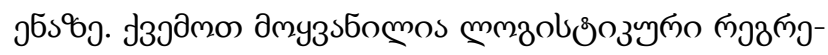

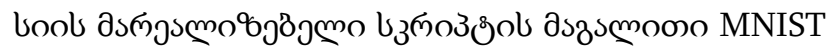

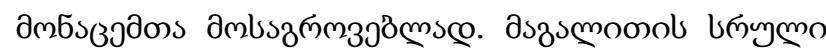

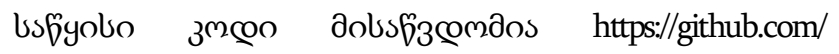

aymericdamien/TensorFlow-Examples/blob/

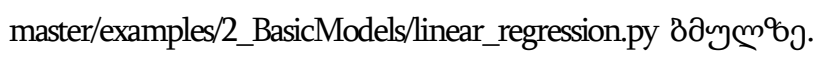

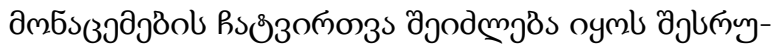

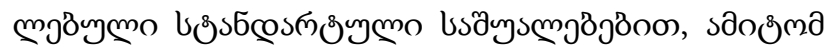

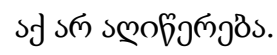

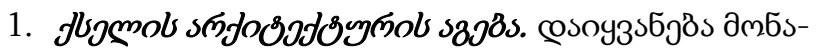

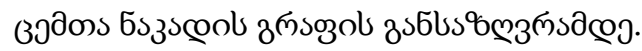

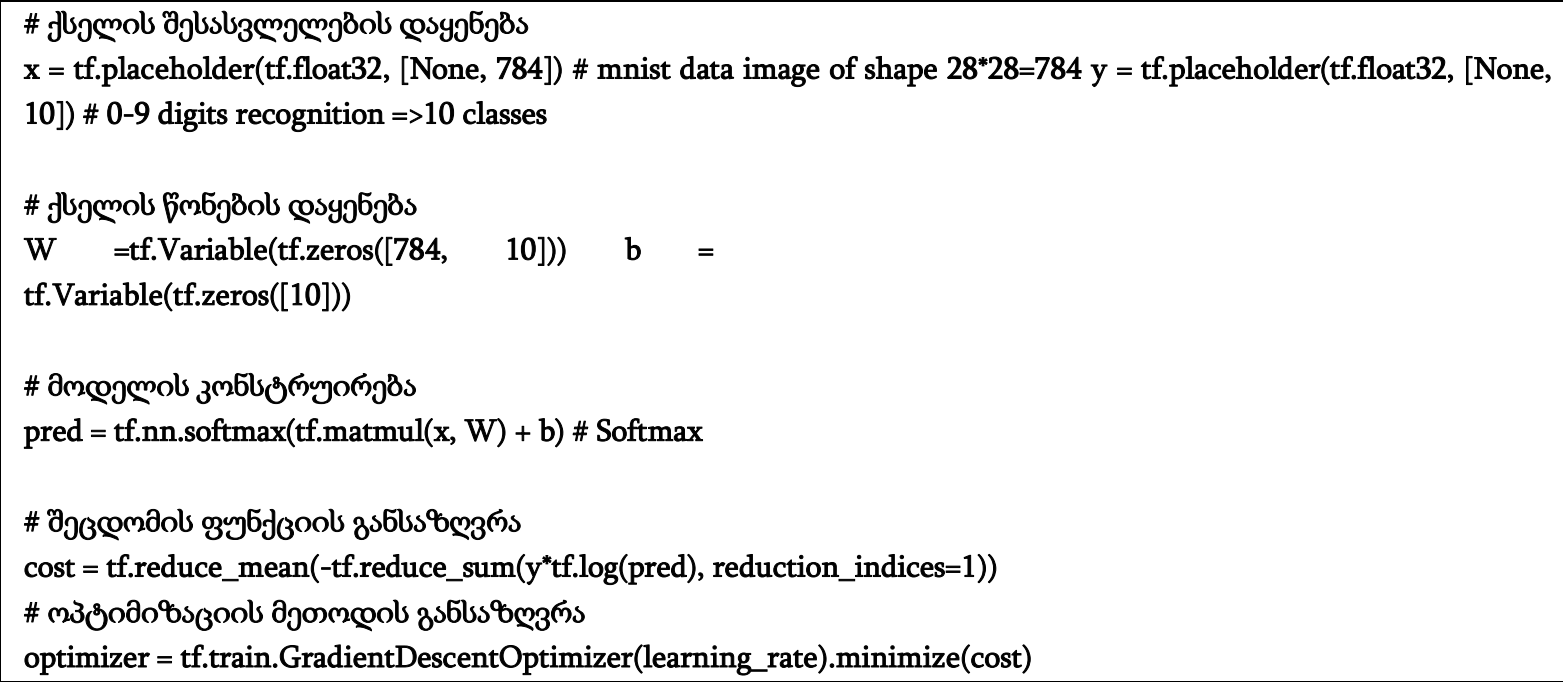




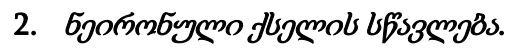

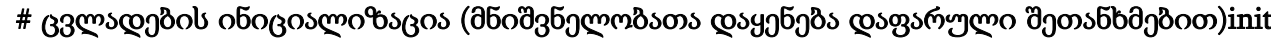

$=$ tf.global_variables_initializer()

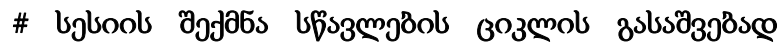

withtf.Session() assess:

\# bjuool ofozosmossosos

sess.run(init)

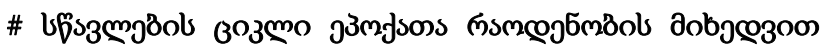

forepochinrange(training_epochs):

avg_cost $=0$.

total_batch = int(mnist.train.num_examples/batch_size)

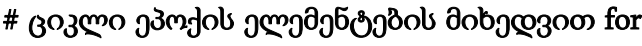

$i$ in range(total_batch):

batch_xs, batch_ys = mnist.train.next_batch(batch_size)

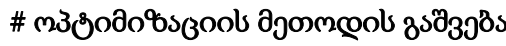

_, c $=$ sess.run $([$ optimizer, cost $]$, feed_dict $=\{x$ : batch_xs,

$\mathrm{y}$ : batch_ys\})

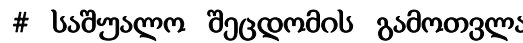

avg_cost $+=$ c / total_batch

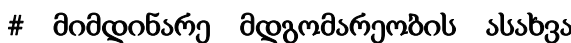

if $($ epoch +1$) \%$ display_step== 0 :

print("Epoch:", '\%04d' \% (epoch+1), "cost=", "\{:.9f\}".format(avg_cost))

print(“Optimization Finished!")

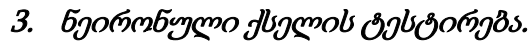

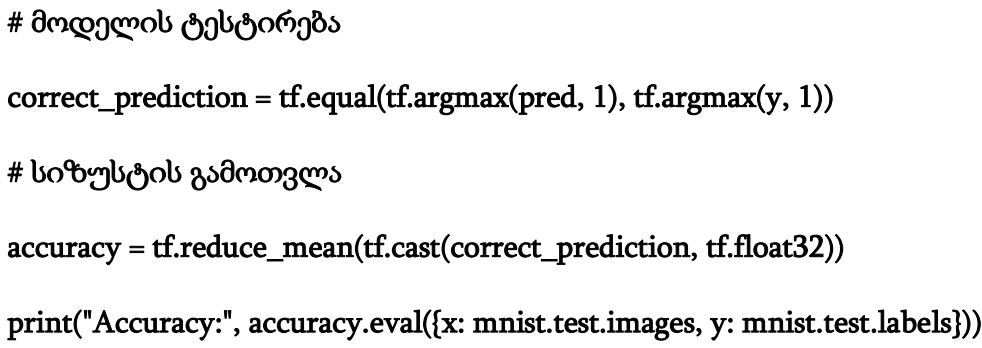

\section{7. ฉовмпмолзз MXNet}

\section{1 дмзмл sмбำ}

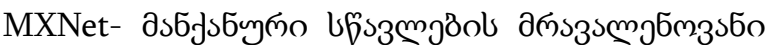

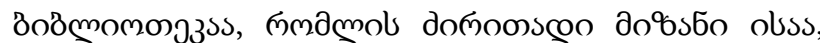

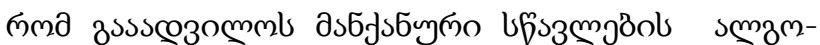

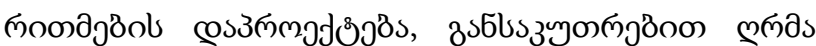

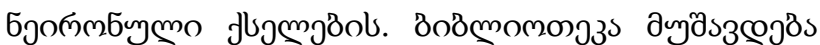

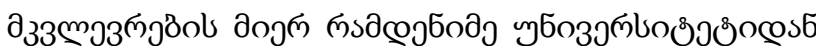


œs зma3sбooœs5 Amazon Web Services, Baidu, Intel,

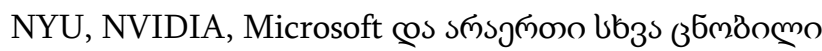

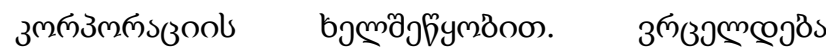
Apache 2.0 mo $395^{6 \% o o n . ~}$

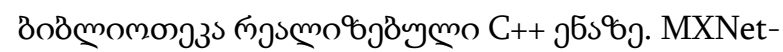

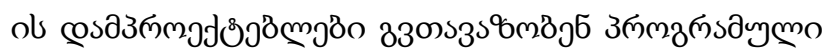

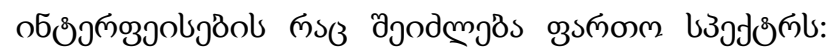
C++, Python, R, Scala, Julia, Perl, Matlab, JavaScript.

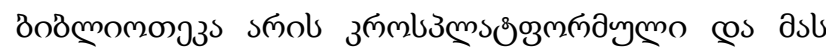

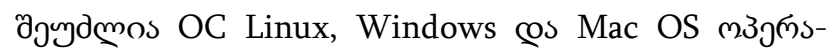

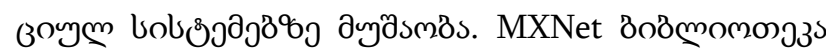

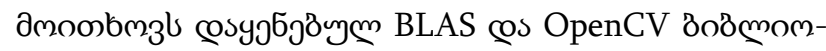

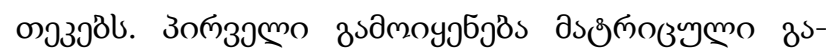

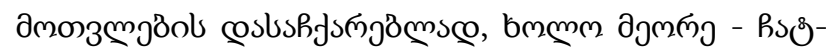

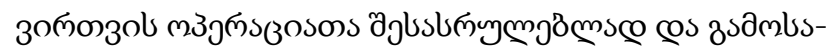

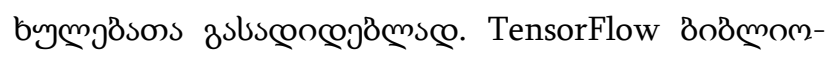

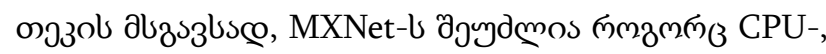

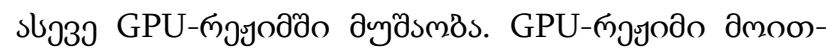

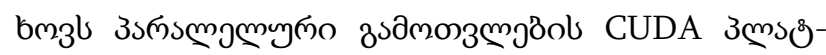

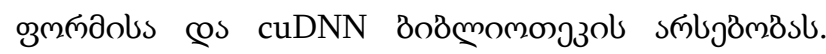

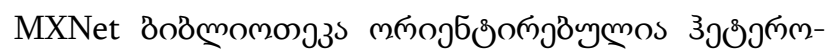

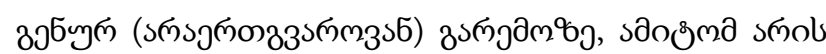

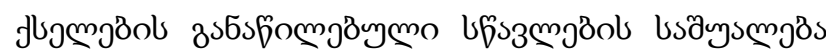

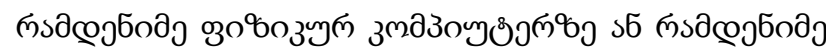

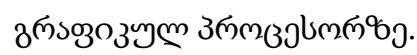

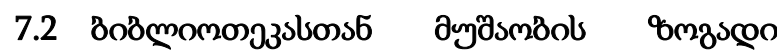

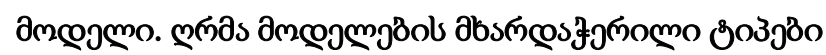

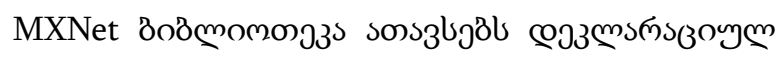

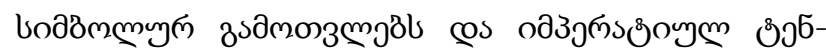

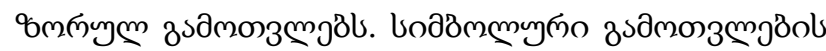

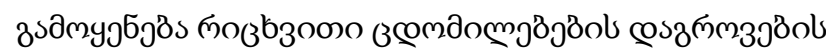

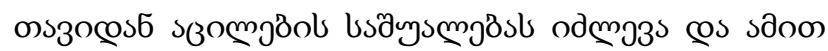

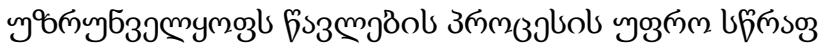

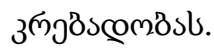

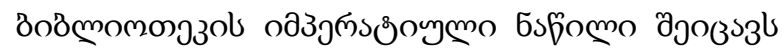

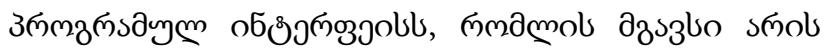

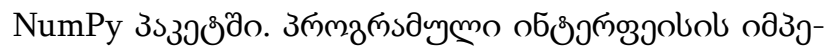

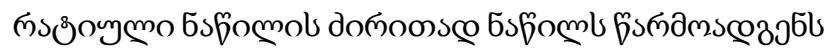

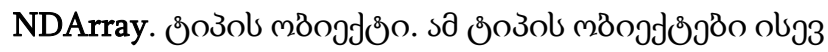

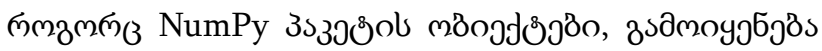

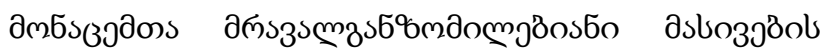

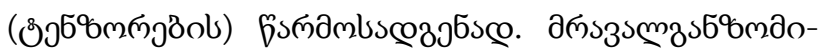

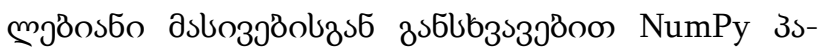

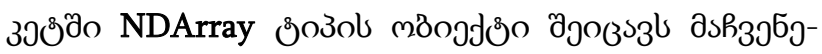

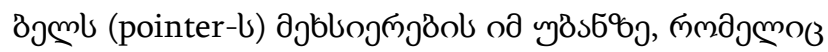

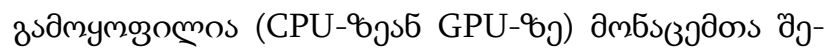

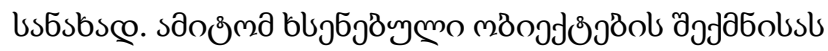

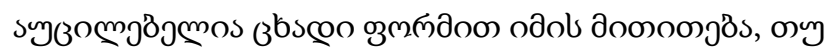

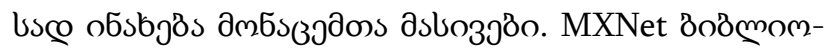

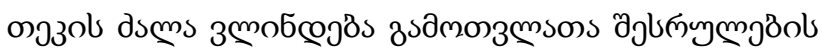

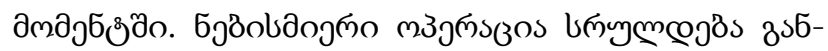

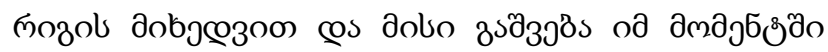

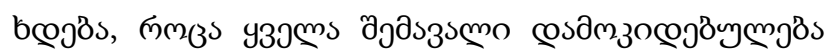

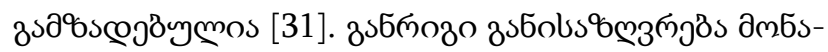

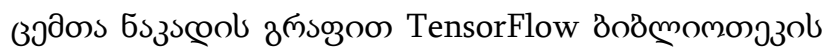
sбsmmzоутыс

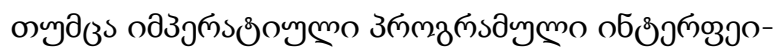

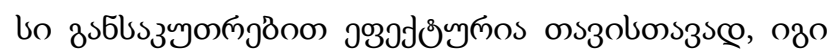

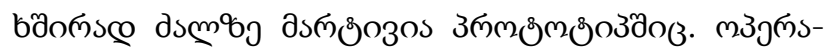

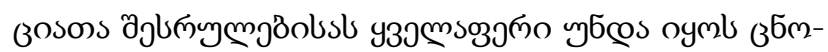

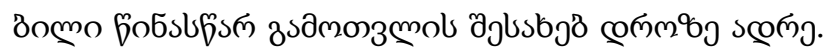

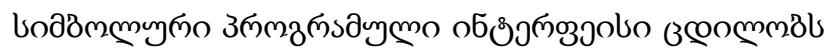

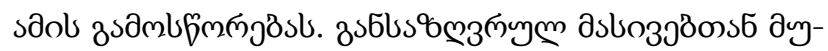

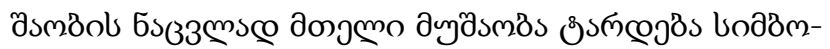




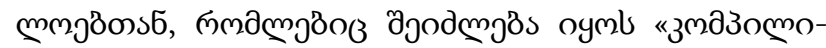

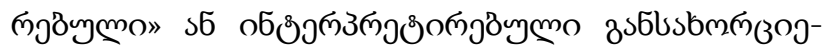

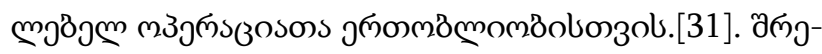

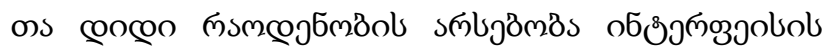

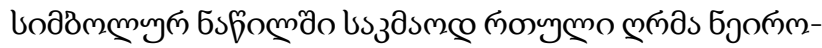

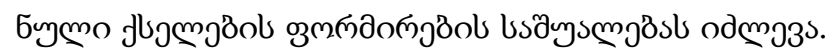

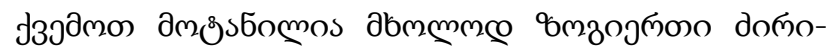

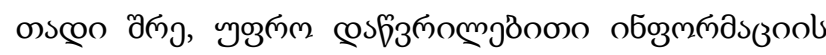

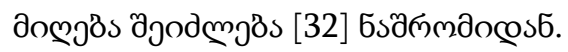

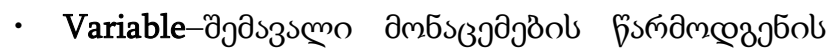
ว๓กว.

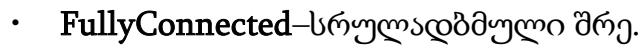

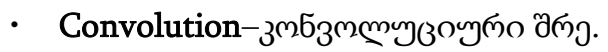

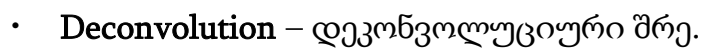

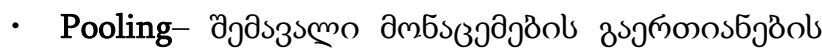

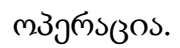

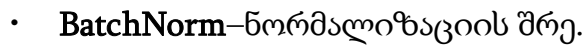

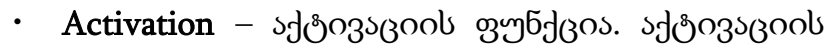

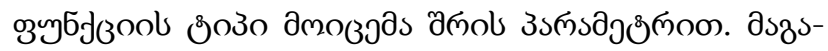
mooföos:"tanh", "relu” @os bb3̧s.

- *Output (aszsmoonsc, SoftmaxOutput, Linear-

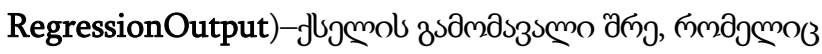

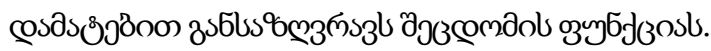

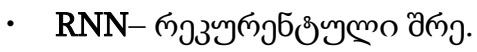

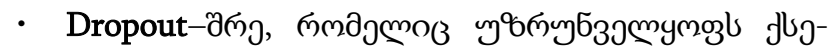

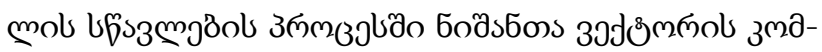

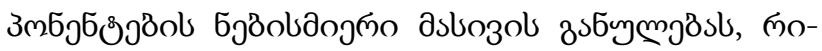

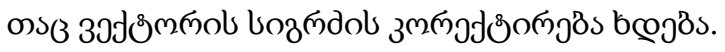

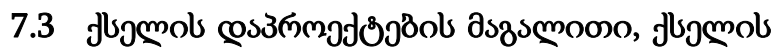

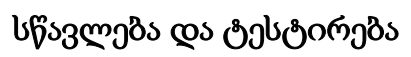

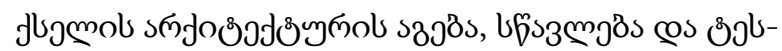

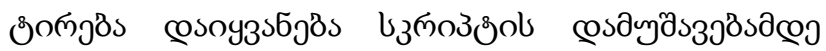
Python jโs\%?.

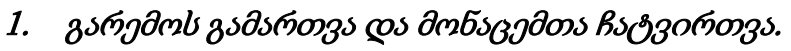

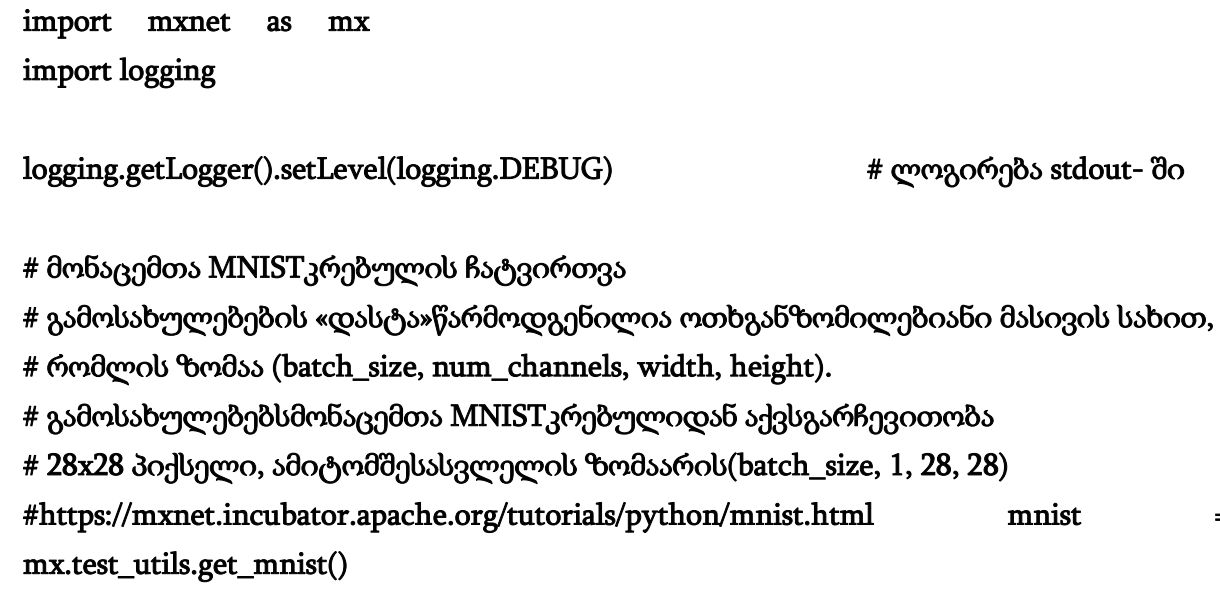




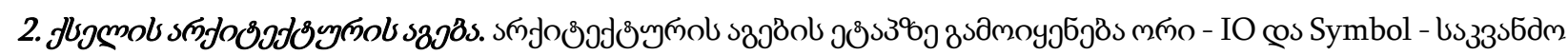
земын. Зоп̆

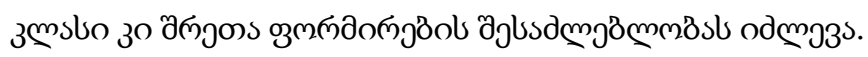

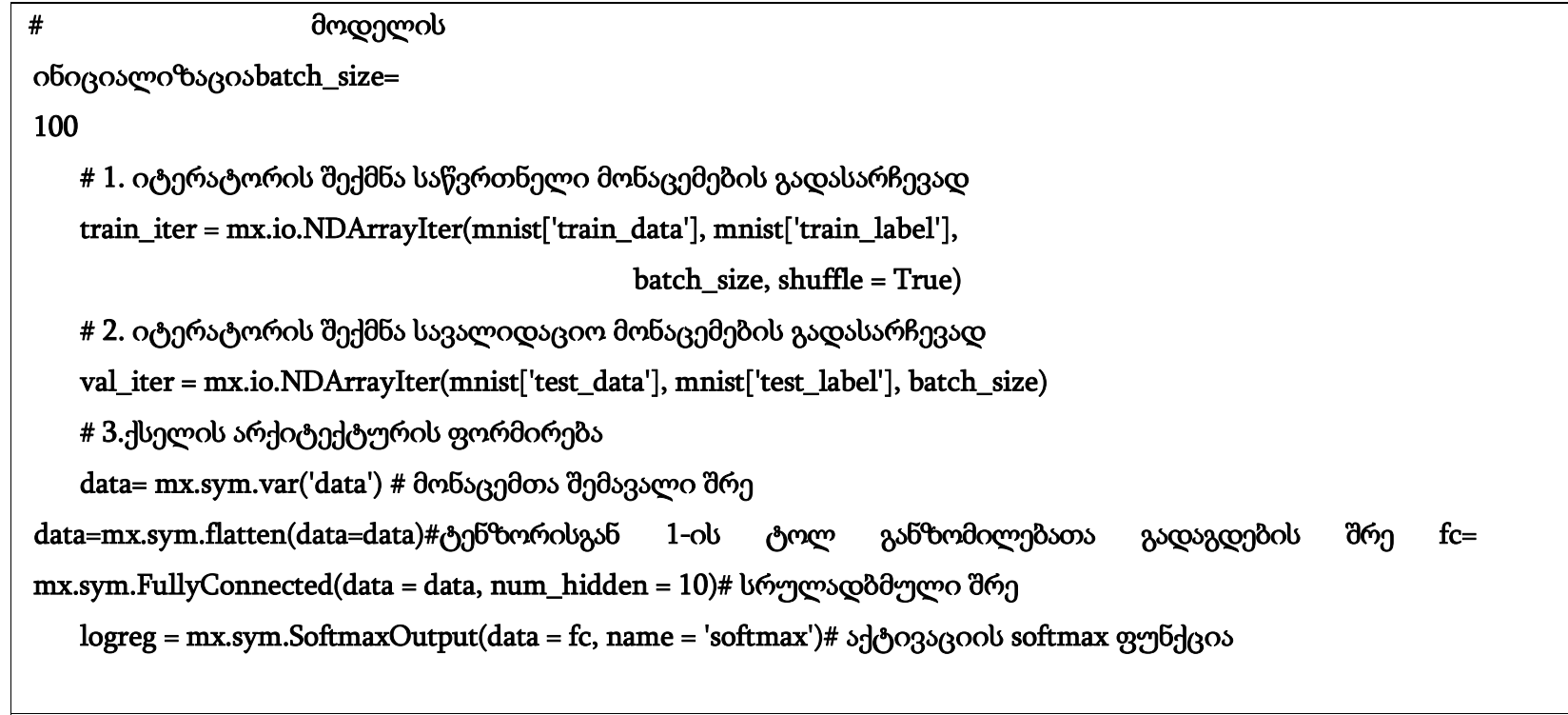

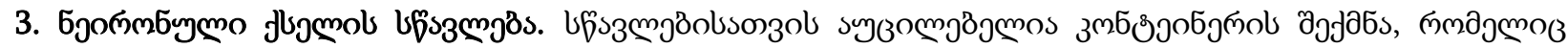

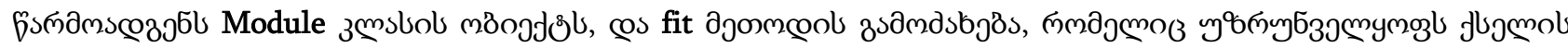

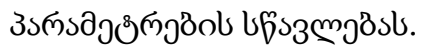

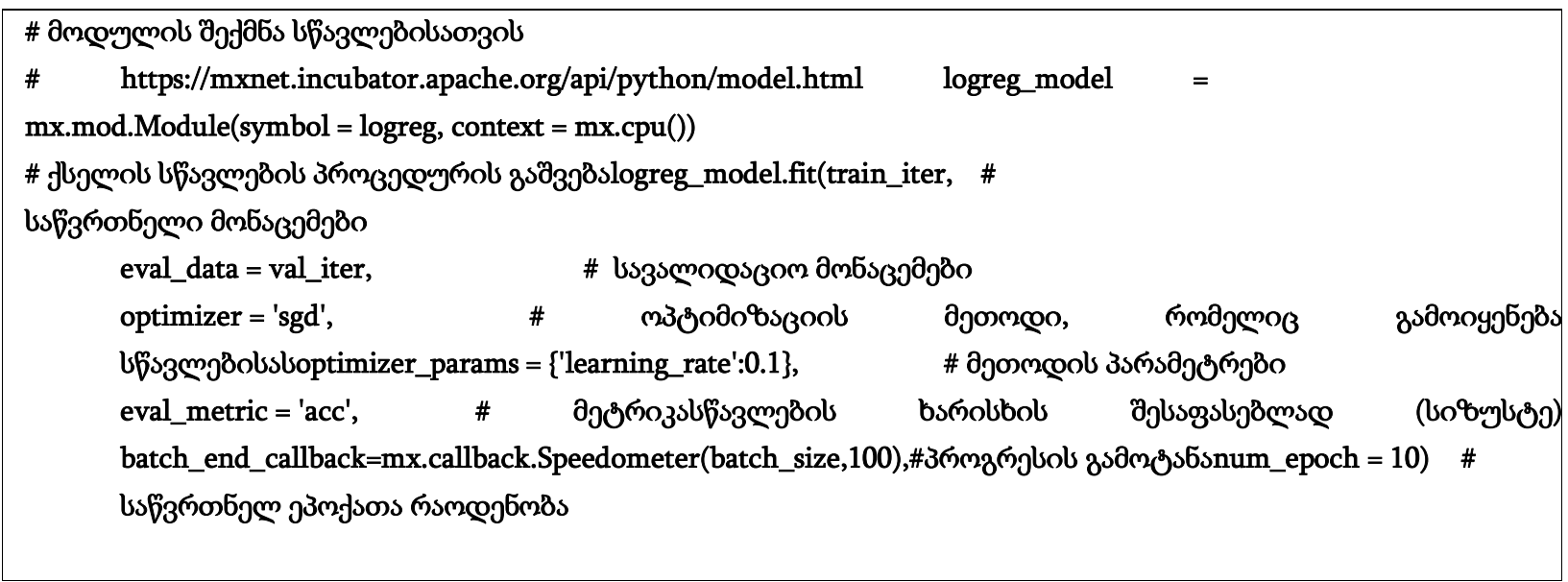

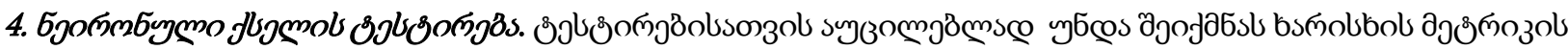

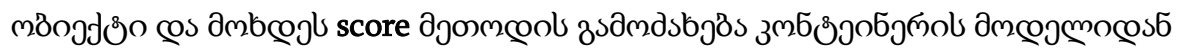




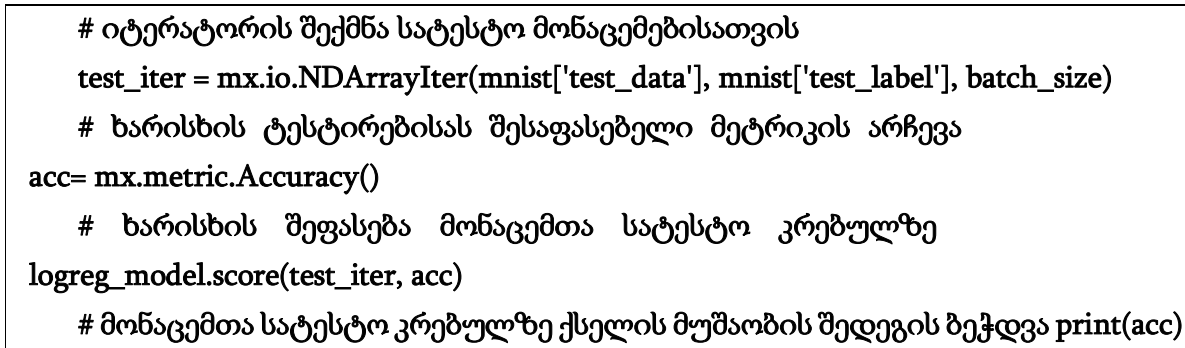

$\cos 333^{6 s}$

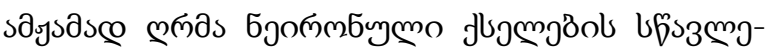

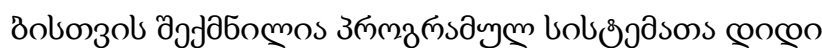

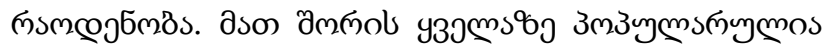
Caffe, Theano, TensorFlow, Torch cos CNTK. Bo8mom-

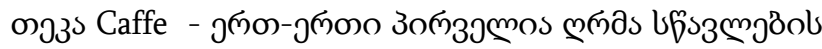

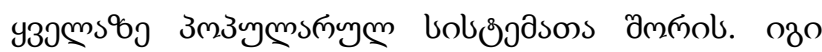

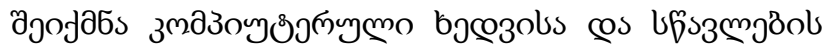

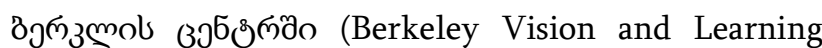

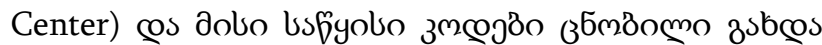

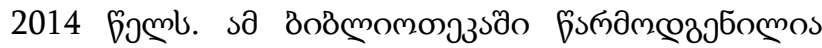

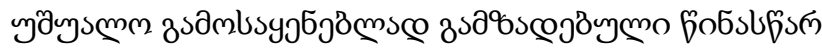

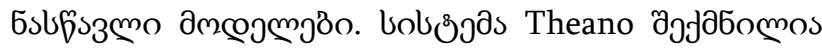

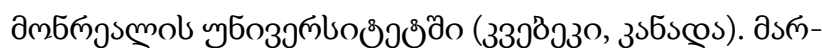

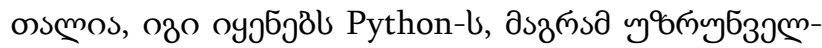

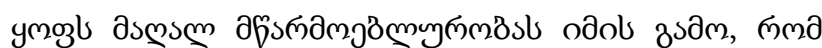

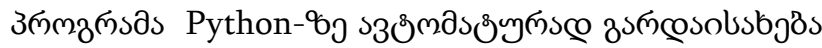

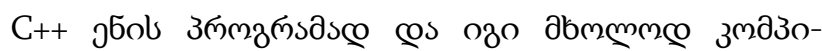

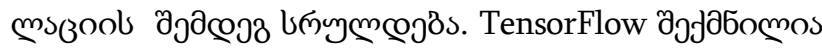

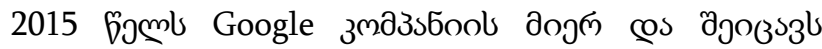

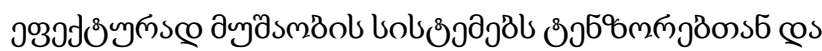

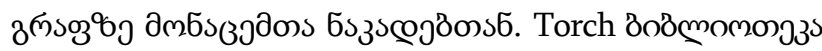

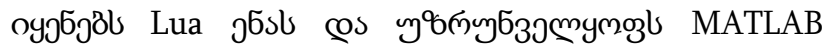

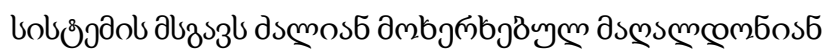

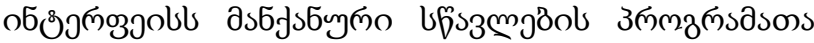

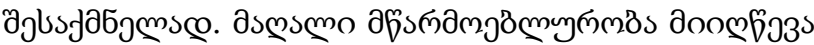

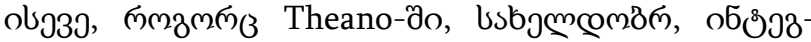

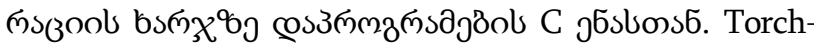

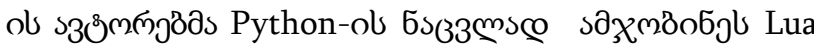

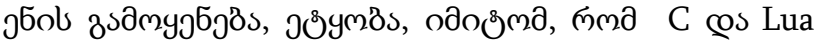

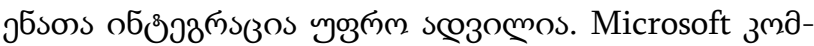

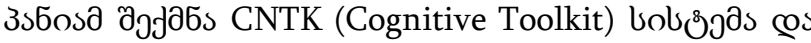

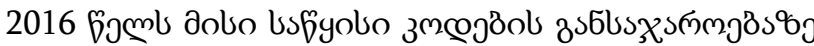

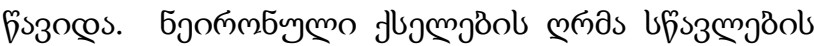

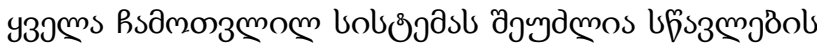

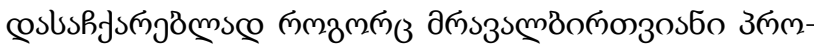

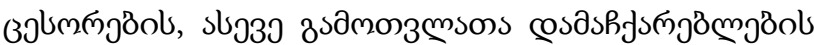

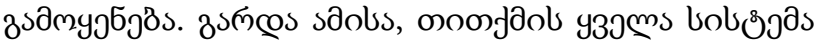

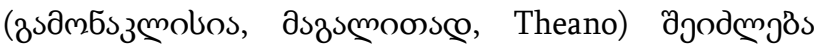

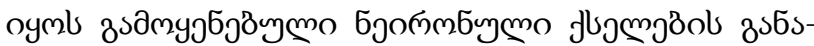

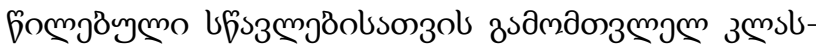

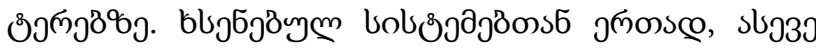

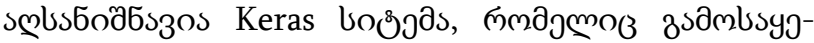

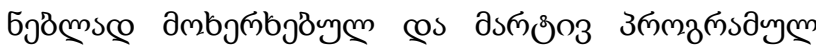

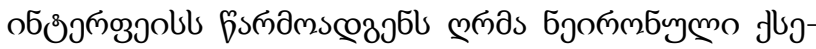

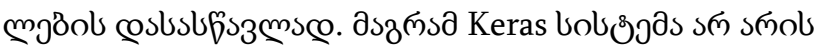

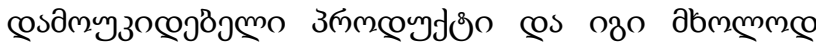




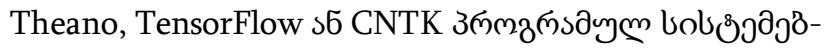

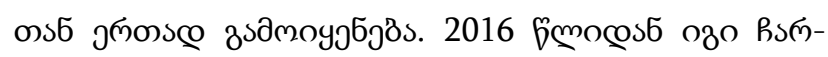

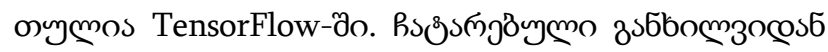

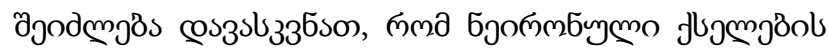

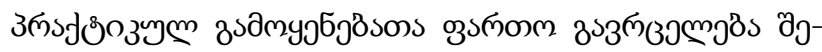

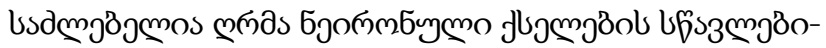

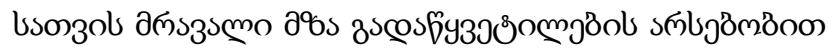

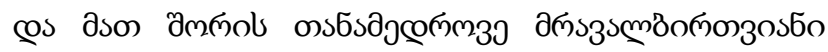

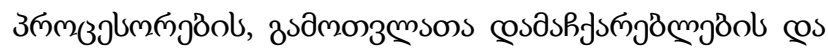

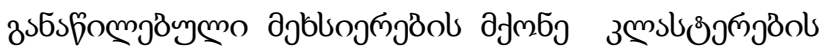
วмภววœวठ̊กо.

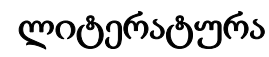

1. Haykin S. Neural networks: Acomprehensive foundation. 2nd edition. Prentice-Hall. 1998, 842 p.

2. Osovsky S. Neural networks for information processing. Finance and statistics. Moscow. 2002, 344 p. (in Russian).

3. Goodfellow I., Bengio Y., Courville A. Deep learning. MIT Press. 2016.

URL: http://www.deeplearningbook.org

4. Tarkov M. Electronic course "Neurocomputer systems".

URL: http://www.intuit.ru/studies/courses/61/61/info (in Russian).

5. Kustikova V.D., Druzhkov P.N. A survey of deep learning methods and software for image classification and object detection. Proceedings of the ORGW 2014. University of Koblenz-Landau. Koblenz. 2014.

6. Kruchinin D.A., Dolotov E.A., Kustikova V.D., Kornyakov K.V., Druzhkov P.N. Comparison of deep learning libraries on the problem of handwritten digit classification. Communications in computer and information science. 2015, 399-411 pp.

7. URL: http://caffe.berkeleyvision.org

8. URL: http://torch.ch

9. URL: https://www.tensorflow.org

10. URL: https://mxnet.apache.org/

11. URL: https://github.com/NervanaSystems/neon

12. URL: https://pytorch.org

13. URL: http://www.iro.umontreal.ca/ lisa/pointeurs/theano_scipy2010.pdf

14. URL: http://deeplearning.net/software/theano/

15. URL: https://keras.io

16. URL: https://github.com/nitishsrivastava/deepnet

17. URL: https://github.com/kyunghyuncho/deepmat

18.URL: https://cran.r-project.org/web/packages/darch/index.html

19. URL:http://hpceducation.unn.ru/\%D0\%BE\%D0\%B1\%D1\%83\%D1\%87\%D0\%B5\%D0\%BD\%D0\%B8\%D0\%B5/ $\%$ D0\%BA\%D1\%83\%D1\%80\%D1\%81\%D1\%8B/intel-neon-framework (in Russian).

20. URL: http://yann.lecun.com/exdb/mnist

21.URL: http://www.cad.zju.edu.cn/home/dengcai/Data/MNIST/images.html

22. Kruchinin D, Dolotov E, Kustikova V, Druzhkov P, Kornyakov K. Comparison of deep learning libraries on the example of the task of classifying handwritten figures.

URL: https://habrahabr.ru/company/intel/blog/254747 (in Russian).

23. URL: https://github.com/BVLC/caffe/tree/windows

24. URL: http://caffe.berkeleyvision.org/tutorial/layers.html

25. URL: http://caffe.berkeleyvision.org/tutorial/solver.html

26. URL: https://github.com/torch/nn/blob/master/README.md

27.URL: https://github.com/torch/nn/blob/master/doc/simple.md\#nn.simplelayers.dok

ISSN 1512-0996

www.shromebi.gtu.ge 
28. URL: https://github.com/torch/nn/blob/master/doc/convolution.md\#nn.convlayers.dok

29. URL: https://github.com/torch/nn/blob/master/doc/transfer.md\#nn.transfer.dok

30. URL: https://github.com/tensorflow/tensorflow

31. TensorFlow: Large-scale machine learning on heterogeneous distributed systems. Preliminary white paper. 2015 URL: http://download.tensorflow.org/paper/whitepaper2015.pdf

32. URL: https://indico.io/blog/getting-started-with-mxnet

\title{
UDC 004.9
}

\section{SCOPUS CODE 1705}

\section{Review of deep learning libraries}

\author{
Archil Prangishvili $\quad$ Department of Computer Engineering, Georgian Technical University, 77 M. Kostava \\ str., 0160 Tbilisi, Georgia \\ E-mail: a_prangi@gtu.ge \\ Oleg Namicheishvili Department of Computer Engineering, Georgian Technical University, 77 M. Kostava \\ str., 0160 Tbilisi, Georgia \\ E-mail: o.namicheishvili@gtu.ge \\ Mikhael Ramazashvili Department of Computer Engineering, Georgian Technical University, 77 M. Kostava \\ str., 0160 Tbilisi, Georgia \\ E-mail: m.ramazashvili@gtu.ge
}

\section{Reviewers:}

Z. Gasitashvili, Professor, Faculty of Informatics and Control Systems, GTU

E-mail: zur_gas@gtu.ge

N. Mchedlisvili, Professor, Faculty of Informatics and Control Systems, GTU

E-mail: galoba47@mail.ru

Abstract. A survey of existing deep learning libraries is given, the task of recognizing handwritten numbers is considered and the network structure that corresponds to logistic regression is described. The article reviews brief descriptions for Caffe, Torch, TensorFlow, and MXNet libraries; a general model for working with the library; supported types of deep models; examples of network development as well as training and network testing.

Key words: Caffe; convolutional layers; handwritten digit classification; image classification; machine learning; MXNet; neural network; object detection; Python; TensorFlow; Torch. 


\title{
UDC 004.9
}

SCOPUS CODE 1705

\section{Обзор библиотек глубокого обучения}

Арчил Прангишвили Департамент компьютерной инженерии, Грузинский технический университет, Грузия, 0160, Тбилиси, ул. М. Костава, 77

E-mail: a_prangi@gtu.ge

Олег Намичейшвили Департамент компьютерной инженерии, Грузинский технический университет, Грузия, 0160, Тбилиси, ул. М. Костава, 77

E-mail: o.namicheishvili@gtu.ge

Михаил Рамазашвили Департамент компьютерной инженерии, Грузинский технический университет, Грузия, 0160, Тбилиси, ул. М. Костава, 77

E-mail: m.ramazashvili@gtu.ge

\section{Рецензенты:}

3. Гаситашвили, профессор факультета информатики и систем управления ГТУ

E-mail: zur_gas@gtu.ge

Н. Мчедлишвили, профессор факультета информатики и систем управления ГТУ

E-mail: galoba47@mail.ru

Аннотация. Дан обзор существующих библиотек глубокого обучения, рассмотрена задача распознавания рукописных цифр и описана сетевая структура, которая соответствует логистической регрессии. Для библиотек Caffe, Torch, TensorFlowи MXNet даны краткие описания, общая модель работы с библиотекой, поддерживаемые типы глубоких моделей, примеры разработки сети, обучение и тестирование сети.

Ключевые слова: Caffe; Torch; TensorFlow; MXNet; нейронная сеть; свёрточные слои; обучающая машина; язык Pуthon; классификация рукописных цифр; классификация изображений; обнаружение объекта.

\author{
8s6bomzol onsळомо 05.03.2019

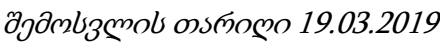

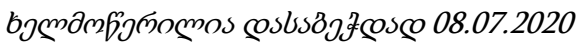

